wird noch vieler Arbeit bedürfen, um hier zu sicheren Schlüssen zu gelangen, vor allem die Untersuchung von Krebslebern selbst.

Nicht mit Unrecht kann man gegen die Untersuchung von Leichenorganen den Einwand erheben, daß man nicht wissen könne, wie weit die stets vorhandenen Fäulnisvorgänge an den Ergebnissen beteiligt sind. Gewiß stellen sie eine sehr unerwünschte Komplikation dar, aber sie betreffen sämtliche Fälle, und es liegt kein Grund vor, ihnen in speziellen Fällen einen besonders großen Umfang zuzuschreiben. Die Möglichkeit, daß der Zufall an dem Ergebnis beteiligt ist, wird man allerdings trotzdem zugeben müssen, sie wird sich nur durch sehr große Untersuchungs-. reihen ausschließen lassen.

Auf die sonst noch erhobenen Zahlen - abgesehen vom Milchsäurebefund - . soll hier nicht eingegangen werden: sie werden vielleicht später im Zusammenhang: mit weiteren Untersuchungen Verwertung finden, als bemerkenswert möchte ich nur die Konstanz des Aschegehaltes in den 10 untersuchten Fällen erwähnen.

\title{
XXXII.
}

\section{Hämatologische Studien}

(III. Fortsetzung zu Bd. 143 u. 174.)

\section{Leukozyten und Leukämie.}

Von

Prof. E. N e u m a $n$, Königsberg.

Seit der Veröffentlichuing meiner ersten Abhandlungen über eine myelogene Leukämie (Arch. d. Heilk. von E. W a g n e r, Bd. 11 u. 13, 1870 u. 1872) haben zwar alle späteren Untersucher bestätigt, daß dem Knochenmark eine hervorragende Bedeutung für die Entstehung der leukämischen Blutbeschaffenheit zukommt, doch hat sich unter dem Einfiuß der durch Ehrlich geschaffenen verfeinerten. Technik der Blutuntersuchung eine von der meinigen abweichende Auffassung der Beteiligung dieses Organs geltend gemacht. Die Rolle, welche ich ihm zuschrieb, wurde auf ein sogenanntes „myeloides Gewebe“ (,myeloides System", „myeloiden Apparat", ,myeloides Parenehym") übertragen; das Knochenmark soll zwar den Hauptrepräsentanten desselben darstellen, aber auch andern Organen werden ,,myeloide" Bestandteile zugeschrieben, und es wird ferner dem „myeloiden Gewebe" als g l e i c h w e r t i g für die Pathogenese der Leukämie ein gleichfalls im Körper über verschiedene Organe verbreitetes "lymphatisches Gewebe" (,lymphatisches System") an die Seite gestellt.

Ähnlich wie V i r c h o w, der nur Milz und Lymphdrüsen (inklusive Lymphfollikel) als Quelle der farblosen Blutzellen kannte, eine lienale und eine lymphatische Form der Leukämie unterschied, wird gegenwärtig gelehrt, daß es einen doppelten Ursprung der Leukämie gebe: aus dem myeloiden System und aus dem lymphati- 
schen System, und daß die Leukämie also keine Organerkrankung, sondern eine „Systemerkrankung“ sei. Es gründet sich diese Lehre bekanntlich hauptsächlich darauf, daß $\mathrm{Ehrlich}$ glaubte nachweisen zu können, daß die farblosen Zellen. des Blutes zwei ,spezifisch" verschiedene Zellstämme umfassen, welche aus zwei gleichfalls ,spezifisch" verschiedenen Gewebstypen, dem Myeloid- und dem lymphatischen Gewebe, in das Blut gelangen und deren Nachweis im leukämischen Blute genügen würde, um den Sitz des Krankheitsherdes in dem einen oder dem andern dieser Gewebe erkennen zu lassen.

Auch in dieser Aufrechterhaltung der Bedeutung des mikroskopischen Blutbefundes für die Differentialdiagnose zwischen zwei durch ihren Ursprung sich unterscheidenden Arten der Leukämie liegt einerseits eine thbereinstimmung mit der alten Vir e h ow schen Auffassung, nach welcher ebenfalls von der Qualität der Zellen des leukämischen Blutes Aufschluß darüber erwartet wurde, ob es sich um eine lienale oder lymphatische Leukämie handle, andrerseits ein Gegensatz zu der Ansicht, zu der ich gelangt war, daß nämlich die Blutuntersuchung keinen Rüekschluß auf den Krankheitsherd gestatte.

So verbreitet die Ehrli e h sche Lehre ist, hat es ihr doch nicht an Gegnern gefehlt; ich brauche nur hinzuweisen auf die Arbeiten von $W_{a} z^{1}$ ) und auf die des kürzlich verstorbenen, insbesondere auf dem Gebiete der Hämatologie verdienten Klinikers Ernst Grawitz ${ }^{2}$ ), beide haben in bezug auf die Leukämie im wesentlichen den von mir ausgesprochenen Grundsätzen beigestimmt. Eine starke Opposition gegen die E h r l i c h sche Lehre von der Spezifität zweier versehiedener Gruppen von Leukozyten hat sich ferner bei den Anatomen bemerkbar gemacht, welche großenteils an der unitarischen Auffassung dieser Zellen festhalten; in neuester Zeit sind es namentlich $W$ eide $\mathrm{nreich}{ }^{3}$ ) und $\mathrm{Maximow}$ ) gewesen, welche in ihren das Problem nach allen Richtungen hin erfassenden ausführlichen Arbeiten der Ablehnung der behaupteten spezifischen Verschiedenheiten der Leukozyten einen sehr entschiedenen Ausdruck geliehen und dadurch auch den Boden, auf welchem die Einteilung der Leukämien nach E h r li c h schem Prinzip aufgebaut ist, erschüttert haben.

Bisher haben die sehwerwiegenden Ausführungen der letztgenannten Autoren ebensowenig eine Widerlegung gefunden wie die schon früher von vielen andern, so auch von mir ${ }^{5}$ ) erhobenen Einwände, und es sind die daraus sich ergebenden Konsequenzen für die Theorie der Leukämie noch nicht gezogen worden. Der dualistischen Auffassung der Leukämie ist es infolgedessen gelungen, die Vorherrschaft zu

$\left.{ }^{1}\right) \mathrm{Walz}$, Arbeiten aus dem Tübinger Path. Institut, Februar 1899. - Idem, Zentralblatt f. allg. Path. u. path. Anat. Bd. 12, Nr. 23. 1901.

2) E. Grawitz, Klinische Pathologie des Blutes. 2. Aufl. 1906.

3) Weidenreich, Arch. f. mikr. Anat. Bd. 72, 73. 1908, 1909.

$\left.{ }^{4}\right)$ M axi m ow, ebenda Bd. 73, 76. 1909, 1910.

5) Vgl. Hämatologische Studien II d. Variabilität der Leukozyten, zugleich ein Beitrag zur Entzündungslehre. Virch. Arch. Bd. 174, 1903. 
behaupten, wie ein Blick auf die neueste Leukämieliteratur zeigt; es erscheint daher geboten, nochmals auf die Bedenken zurückzukommen, die sich dieser Auffassung entgegenstellen.

Zunächst einige Worte über die Einführung eines , my eloiden Gew e bes " in die Nomenklatur, die zwar bei Klinikern und Pathologen Anklang gefunden hat, von den Anatomen dagegen nicht rezipiert worden ist. Schon formell muß es ja allerdings unzulässig und absurd erscheinen, das normale Gewebe des roten blutbildenden Markes im ganzen als ,myel o id “, d. h. mark ä hn l i c h zu bezeichnen oder den Ausdruck für eine Komponente des Marks, nämlich für einen durch große granulierte Zellen, die eigentlichen Markzellen, ausgezeichneten Teil desselben im Gegensatze zu einem andern, Lymphozyten enthaltenden lymphatischen Teil zu gebrauchen. Abgesehen hiervon ist es aber auch histologisch nicht richtig, daß das Knochenmark zwei voneinander verschiedene Gewebe in sich vereinigt, es besteht vielmehr ein durchaus ein hei tlich es Gewe be, in welchem Zellen verschiedener Art, größere granulierte Zellen und Lymphozyten in gleicher Weise eingelagert sind, die letzteren meistens zerstreut zwischen ersteren, bisweilen auch (selten!) in dichteren, Follikeln ähnlichen Haufen zusammenliegend. Da diese Lymphozyten übrigens gegen die andern Zellen an Zahl sehr zurücktreten, so erscheint auch, wie schon $W$ al $z$ richtig bemerkt hat, der durch mich in Gebrauch gekommene Namen , lymphoides M ark " für das normale blutbildende rote Mark, welchen ich wählte, um die damals noch unbekannte Verwandtschaft desselben mit den Lymphdrüsen auszudrücken, nicht mehr passend, denn der Lymphozytenbegriff hat sich seitdem allgemein auf kleinere oder größere, jedenfalls ungranulierte Zellen begrenzt. Man reserviert die Bezeichnung besser auf pathologische Fälle mit starker Vermehrung dieser Zellen und sollte sich für den normalen Zustand mit dem alten Terminus technicus: r o tes Mark begnügen; eine Verwechslung mit einem durch Hyperämie oder Extravasate geröteten Fettmark wird ja leicht durch einen Zusatz zu vermeiden sein.

Daß man ebenso wie für das Knochenmark auch für Milz und Lymphdrüsen neuerdings die Existenz eines „myeloiden“ Gewebes neben dem übrigen lymphatischen Parenchym angenommen hat, läßt sich ebenfalls nicht rechtfertigen, denn auch hier bestehen nicht zwei verschiedene Gewebsformationen, sondern nur eine Verschiedenheit der in dasselbe Gewebe eingelagerten Zellen, und die Unterscheidung von zwei besonderen Gewebstypen kann nur zu falschen histologischen Vorstellungen führen. Es sei daher der Vorschlag gestattet, den Ausdruck „,myeloides Gewebe" nur für solche pathologische Fälle zu gebrauchen, wo sich außerhalb des Knochenmarks in anderen Organen ein Gewebe bildet, welches die für das Knochenmark hauptsächlich charakteristische Eigenschaft besitzt, r o te Blutkörperchen aus sich heraus zu erzeugen,

Auch in bezug auf den Ausdruck , Spezifität" sei eine Bemerkung vorausgeschickt, da derselbe nicht immer in gleichem Sinne gebraucht wird. Man 
kann es für die Annahme eines spezifisch versehiedenen Charakters zweier Zellen als genügend ansehen, wenn die direkte Umwandlung einer Zelle in die andere nicht möglich ist; in diesem Sinne werden ziemlich allgemein, obwohl auch in dieser Beziehung eine volle Meinungseinheit nicht vorhanden ist, neutrophile und eosinophile. Leukozyten als zwei infolge vorgeschrittener Differenzierung spezifisch versehiedene Zellarten betrachtet. Für die Dualisten aber gehört zum Spezifitätsbegriff zugleich, daß der Ursprung $z$ weier Zellen nicht auf eine im extrauterinen Leben vorkommende gemeinschaftliche $\mathrm{Sta} \mathrm{mm} \mathrm{zelle} \mathrm{zurückgeführt} \mathrm{werden} \mathrm{kann;} \mathrm{hier} \mathrm{wäre} \mathrm{also}$ auch eine indirekte Umwandlung durch Rückbildung der einen Zelle auf ihre Stammform und progressive Entwicklung letzterer zu dem andern Zelltypus ausgeschlossen. Im folgenden werde ich die Bezeichnung ,spezifisch" nur in letzterem Sinne gebrauchen.

Hiernach gestaltet sich also die für die Leukozyten zu entscheidende Streitfrage so, daß die Unitarier sämtliche Formen derselben auf eine gemeinschaftliche, auch extrauterin stets vorhandene Stammzelle zurückführen, während die Dualisten in ihnen zwei Gruppen unterscheiden, die von zwei selbständigen Stammzellen abzuleiten sind und also nach Ablauf der embryonalen Entwicklung keinen $\mathrm{Zu}$ sammenhang miteinander mehr haben.

Welche Mittel stehen uns nun zu Gelote, um hierüber eine Entscheidung zu treffen? Man könnte wohl vermuten, daß eine gewisse Überschätzung derselben von beiden Seiten stattgefunden hat und daß hierdurch die erforderliche Vorsicht bei der Beurteilung verhindert und der Streit versehärft worden ist. Vielleicht wird eine endgültige Entscheidung erst dann möglich sein, wenn es gelungen sein wird, mit den farblosen Blutzellen dasselbe Experiment anzustellen, welches $\mathrm{R}$ o b e r t $\mathrm{K}$ o $\mathrm{eh}$ mit den Bakterien auszuführen lehrte, nämlich die einzelnen Zellen zu isolieren und ihre Lebensvorgänge längere Zeit hindurch in vitro zu verfolgen. Ebenso wie die Lehre von der Spezifität der Mikroorganismen auf der durch dieses Experiment erwiesenen Möglichkeit einer R e in k u lt u r derselben beruht, würde sich den Dualisten die Aussicht eröfinen, den direkten Beweis für die Richtigkeit ihrer Anschauung von der Spezifität der beiden von ihnen angenommenen Leukozytengruppen zu erbringen. Bis jetzt ist, soviel mir bekannt, die Aufgabe einer "Blutzüchtung" noch von niemand vom dem bezeichneten Gesichtspunkte aus in Angriff genommen worden, alle vorliegenden Beobachtungen über das Schicksal ,,überlebender" Blutzellen entsprechen selbstverständlich diesem Zwecke nicht, doch läßt sich die Hoffnung nicht unterdrücken, daß die Schwierigkeiten der Aufgabe nicht unbesiegbar sind. Carrels glückliche Erfolge mit Gewebszüchtungen haben ja gezeigt, daßj der Ernährungs-, Wachstums- und Proliferationsfähigkeit lebender Teile auch außerhalb des Organismus unter geeigneten Bedingungen eine viel größere Beständigkeit zukommt als bisher bekannt war; zu befürchten bleibt freilich, daß der Versuch an der Unmöglichkeit, eine zur Beobachtung hinreichende Absonderung einzelner lebenden Blutzellen zustande zu bringen, scheitern wird. 
Alle bisherigen Untersuchungen beziehen sich auf die Vorgänge in den Körpergeweben und -flüssigkeiten und die Wege, welche die Forschung eingesehlagen hat, um in die Verhältnisse der Leukozyten zueinander einzudringen, sind verschiedener Art. Einer derselben bestand in der Beobachtung der z e it li che n A u f e in a n d e r f o lge der verschiedenen Leukozytenformen bei ihrer Entwicklung. So wertvoll die auf diese Weise gewonnenen Erfahrungen sind, so läßt sich doch nicht erwarten, daß dieselben zu unzweideutigen Resultaten führen. Wenn wir bei dem Embryo zuerst, wie die Untersuchungen von $\mathrm{S}$ a $\mathrm{x}$ e r, M a i m o w und D a $\mathrm{nt}$ s c h a k of $\mathrm{f}$ lehren, zuerst kleine lymphozytäre Körperchen im Blute auftreten und erst später größere, multinukleäre, granulierte Zellen folgen sehen, so geht daraus zwar mit Bestimmtheit hervor, daß die ersteren nicht aus letzteren entstehen, aber es ist nicht ohne weiteres der Ursprung dieser auf jene zu beziehen, der Schluß: post hoc, ergo propter hoe verbietet sich durch die Überlegung, daß die zuletzt aufgetretenen Zellen sich auch selbständig entwickelt haben können; es bedarf also erst eines Beweises, daß dies nicht der Fall ist. Ebenso wird bei der Entstehung eines normalen oder pathologischen, heterotopen. Knochenkerns mit neugebildetem Knochenmark für die genetische Beziehung der in letzterem auftretenden verschiedenen Markzellen nicht unbedingt die Zeitfolge, in welcher sie erseheinen, maßgebend́ sein, da die Konkurrenz mehrfacher, sukzessive in Wirksamkeit tretenden Ursprungsquellen nicht auszuschließen ist, und wenn ferner im Verlaufe eines entzündlichen Exsudationsprozesses der Charakter der Exsudatzellein sich ändert, so kann dies ebensowohl von einer Umwandlung derselben als auch davon, daß Zellen andern Ursprungs mit der Zeit auftreten, herrühren, stets wird eine Kontrolle, die sich auf eine Entscheidung zwischen dieser Alternative richtet, erforderlich sein.

Nicht weniger werden wir uns unsicher fühlen müssen, wenn wir den Versuch machen, $\mathrm{t}$ o $\mathrm{p} 0 \mathrm{~g} \mathrm{r}$ a $\mathrm{ph}$ i s $\mathrm{ch}$ die Veränderungen zu verfolgen, welche die Leukozyten auf den von ihnen eingeschlagenen Wegen erleiden. Schon auf dem Wege von den Lymphdrüsen zu dem Ductus thoracicus können sich zu den Lymphozyten, welehe aus jenen hervorgegangen sind, Leukozyten aus dem Blute unbestimmten Ursprungs hinzugesellen, was zur Vorsicht bei der Entscheidung darüber, inwieweit eine progressive Entwicklung der Lymphozyten zu andern Zellformationen in der Lymphe stattfindet, mahnt. Dasselbe Bedenken macht sich geltend bei der Untersuchung der Sehicksale der aus Lymphfollikeln stammenden Zellen auf ihrer Wanderung durch das bedeckende Epithel von Schleimhäuten an die Oberfläche, denn auch auf diesem allerdings sehr kurzen Wege ist die Möglichkeit des Hinzutretens von Zellen anderer Art aus dem Blute gegeben und muß bei der Beweisführung berücksichtigt werden.

Die Veränderungen der Leukozyten in der Blutbahn von ihren Ursprungsorten aus zu verfolgen, erscheint vollends aussichtslos; am meisten zu beachten und doch merkwürdigerweise am wenigsten festgestellt dürfte das Verhalten der Leukozyten in den Venen der Blutbildungsorgane (Knochenmark, Milz) im Ver- 
gleich zu den das Parenchym derselben erfüllenden, zum Übergange in das Venenblut bestimmten Zellen sein, doch wäre auch hier wiederum eine Fehlerquelle zu vermeiden, da ja in die Venen auch das durch die Arterien den Organen zugeführte Blut mit seinen Leukozyten gelangt.

In eine dritte Kategorie gehören die Bemühungen, durch die A u s s chaltung eines blutbildenden Organs Aufschluß über die Spezifität der von ihm produzierten Leukozyten zu erhalten, wogegen ich mich schon an anderer Stelle ablehnend ausgesprochen habe. Bekanntlich haben die Ergebnisse von Milzexstirpation en in dem Streite eine Zeitlang eine wichtige Rolle gespielt, und es scheint sogar, daß Ehrlich gerade durch diese Experimente zuerst veranlaßt wurde, sich mit großer Bestimmtheit dahin auszusprechen, daß alle multinukleären Leukozyten des Blutes aus dem Knochenmark stammen und daß sie von den kleineren, einkernigen Lymphozyten spezifisch verschieden wären. Einer solchen Schlußfolgerung stehen aber große Bedenken entgegen. Welehes auch immer die Folgen eines Ausfalls der Milzfunktion für die morphologische Zusammensetzung des Blutes sein mögen (die Angaben darüber stimmen übrigens durchaus nicht überein) - soviel ist sicher, daß dabei $n$ ich $t$ eine bestimmte Art von Leukozyten aus dem Blute völlig verschwindet, und doch wäre es nur in diesem Falle positiv erwiesen, daß diese Zellen von den Zellen anderer blutbildenden Organe spezifisch verschieden und durch letztere nicht zu ersetzen sind; handelt es sich dagegen immer nur — wie es in der Tat der Fall ist - um eine mehr oder weniger beträchtliche Verschiebung der normalen Zahlenverhältnisse der verschiedenen farblosen Blutzellen, so bleiben die gegenseitigen Beziehungen derselben unaufgeklärt, und selbst diese Verschiebungen werden nicht als unmittelbare Folge des Aufhörens der Zufuhr aus der Milz aufzufassen sein, da zugleich mit einer regulierenden Abänderung der normalen Funktion der übrigen blutbildenden Organe zu rechnen ist. Bei der durch Atrophie und andere pathologische Zustände der Milz bedingten Aufhebung der Milzfunktion verhält es sich natürlich nicht anders.

Dieselben Erwägungen würden sich ferner aufdrängen, wenn es möglich wäre, den lymphatischen Apparat oder das Knochenmark gänzlich aus dem Körper zu entfernen. Bei ersterem hat man die Exstirpation durch Anlegung einer Ductus-thoracicus-Fistel zu ersetzen gesucht ( B i edI und D e caste 110 ); wie zu erwarten, waren auch hierdurch nur quantitative Veränderungen in der leukozytären Zusammensetzung des Blutes zu erreichen, und das war auch das Ergebnis der Blutuntersuchungen in pathologischen Fällen von Destruktion der Drüsen, so daß auch hier per exclusionem weder über die Beschaffenheit der Zellen, welche das Blut aus dieser Quelle bezieht, noch über die Spezifität derselben ein genügender Aufschluß zu gewinnen war, ganz abgesehen davon, daß Reste funktionierender Drüsensubstanz wohl ausnahmslos zurückbleiben. - Beispiele für einen vollständigen Ausfall des $\mathrm{Kn}$ o che n marks gibt es nicht, experimentell ist er nicht erreichbar und pathologisch-anatomisch noch nicht beobachtet; die Diskussionen über die Folgen einer fehlenden Zufuhr von Zellen 
aus dem Knochenmark, welche sich an Fälle von Osteosklerose oder andere ausgedehnte Erkrankungen dieses Organs geknüpft haben, zeigen aber, daß auch hier stets alle dem Blute zukommenden Elemente vertreten, keines ganz verschwunden war, so daß daraus nur hervorgeht, daß es keine Zellen gibt, welche ausschließlich vom Knochenmark produziert werden, es gilt dies bemerkenswerterweise auch von den von Ehrlich unbedingt für das Knochenmark in Anspruch genommenen multinukleären Leukozyten, deren Zahl in jenen Beobachtungen nicht einmal eine Verminderung erfahren hatte; die Frage nach der Spezifität dieser Zellen bleibt dabei offen.

Nachdem wir somit die bezeichneten Mittel, letzterer Frage näherzutreten, als ungenügend kennen gelernt haben, sehen wir uns fast ausschließlich darauf angewiesen, nach Überg ang s ormen zu suchen, wie ja in der Tat die Untersuchungen hauptsächlich darauf gerichtet gewesen sind, $\mathrm{zu}$ ermitteln, ob in bezug auf Größe, Struktur und chemische Reaktionen Übergänge zwischen den einzelnen Zellarten festzustellen sind. Eine gewisse Skepsis wird man freilich auch hier walten lassen müssen, da einerseits wirklich vorhandene Übergangsstadien übersehen werden können, falls dieselben schnell vorübergehend sind oder nur an bestimmten Orten aufgefunden werden können, andrerseits aber auch Übergangsformen vorgetäuscht werden können, wenn wir zwei uns mit unsern Hilfsmitteln identisch erscheinende Zellen nicht als voneinander verschieden zu erkennen vermögen und sie demnach ein und derselben Entwicklungsreihe einfügen. Nur eine mit Berücksichtigung dieser Fehlerquellen gleichzeitig am natürlichen und verschiedentlich präparierten Objekte ausgeführteUntersuchung kann zu brauchbaren Ergebnissen führen. Als Material für diese Untersuchungen bieten sich natürlich dar das Blut, die Lymphe und vor allem die Blutbildungsorgane.

Utber die verschiedenen Formen der Blutleukozyten liegen bereits aus älterer

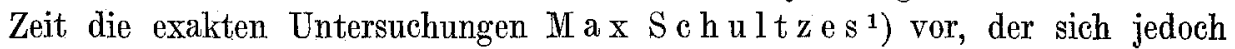
einer Äußerung über die gegenseitigen Beziehungen derselben fast ganz enthalten hat.

M. $S \mathrm{chultze}$ unterschied nach seinen am frischen, seinen natürlichen Zustand darbietenden Blutstropfen angestellten Beobachtungen vier Arten: 1. kleinste Formen mit grofem, kugeligem Kern, geringem Protoplasma, das leichte Trübung, meistens aber keine erkennbaren Körnchen zeigt; 2. etwas größere Formen, deren Kern von demselben Kaliber wie bei 1., deren Protoplasma aber ansehnlich, feingranuliert und im Gegensatze zu 1. amöboid, wenn auch nur träge beweglich ist; 3. t y p i s ch e Forme n : größere Zellen mit feinkörnigem, außerdem kleinste Vakuolen einschließendem Protoplasma, welches in frischem Zustande einen oder zwei, auch wohl mehr Kerne durehschimmern läßt und lebhaft beweglich ist; 4. grobgranulierte größere Zellen mit einem oder zwei dentlichen Kernen, ebenfalls lebhaft beweglich. 'Utbergänge zwischen 3. und 4. sollen vorkommen durch Kombination feiner Körnehen und gröberer Granula.

Die neuere, von $\mathrm{Ehrlich}{ }^{2}$ ) durch seine bekannten Fixierungs- und Färbungsmethoden begründete Einteilung der farblosen Blutzellen hat offenbar mit der M. S chultze schen eine sehr große Ähnlichkeit; wir unterscheiden nach Ehrlichscher Nomenklatur:

1) M. Schultze, Arch. f. mikr. Anat. Bd. 1, 1865.

$\left.{ }^{2}\right)$ Ehrlich-Lazarus, Die Anämie 1898 (in Nothnagels Spez. Path. u. Ther. Bd. 8)

Virchows Arohiv f. pathol, Anat. Bd, 207. Hit. 3. 
1. I y $\mathrm{mph} \mathrm{o}$ z y t e $\mathrm{n}$ mit rundem Kerm und schmälerem oder breiterem, homogen erscheinendem, stark basophilem Protoplasmașaum; 2. gr o Be unin u $\mathrm{k}$ l är e Z elle n mit großem, blassem, ovalem Kern und homogenem Protoplasma und sogenannten U̇ b e r g a $\mathbf{n} \mathbf{g} \mathbf{s}$ formen mit eingeschnürtem großen Kern, das schwach basophile Protoplasma gleichfalls meistens homogen, bisweilen mit spärlichen neutrophilen Granulationen versehen; 3. n e u t r o $\mathrm{ph}$ il e $\mathrm{Z}$ e $l 1$ e $\mathrm{n}$ mit polymorphem Kern und reichlichen neutrophilen Granulationen; 4. e o s in ophile Zellen gleichfalls mit polymorphem Kern und groben, eosinophilen Granulationen; 5. spärliche Mastzellen, ausgezeichnet durch basophile Granulationen.

Durch die Untersuchungen Weidenreic $\mathrm{h} \mathrm{s}^{1}$ ) ist ferner neuestens festgestellt, daß sich außer den genannten Zellen im normalen Blute nicht nur polymorphkernige, sondern auch e i $n$ k e rni g e neutrophile Leukozyten und ebenfalls e in k e rnige eosinophile Zellen, sogenannte „neutrophile Myelozyten“ und „eosinophile Myelozyten“ befinden, beide allerdings nur sehr vereinzelt.

Ttberblicken wir nun die hier aufgezählten Zellformen, so ist es begreiflich, daß man früher ganz allgemein in ihnen eine kontinuierliche Reihe von Formen als Ausdruck einer von den kleinen, einkernigen Lymphozyten bis zur Bildung der größeren, granulierten, polymorphkernigen Zellen vorschreitenden Entwicklung vor sich zu haben glaubte. Auch gegenwärtig besteht darin kaum eine Meinungsverschiedenheit, daß zwischen den großen granulationslosen uninukleären Zellen Ehrlichs und den neutrophilen bzw. eosinophilen Zellen alle Übergänge zu beobachten sind, welche zu der Annahme einer Entwicklung der letzteren aus ersteren zwingen. Der Streit dreht sich nur um das Verhältnis zwischen Lymphozyten und großen uninukleären Zellen Ehrlich scher Nomenklatur.

Ehrlich machte zwisehen beiden einen Strich, indem er sagte, daß, ,die Lymphozyten in ihrer ganzen Erscheinungsform durchaus von dem Typus der großen uninukleären Zellen abweichen und Übergänge zwischen beiden nicht zu beobachten sind". Ein Widerspruch gegen diese so kategorisch hingestellte Behauptung konnte nicht ausbleiben. Schon die Beschreibung, welche Ehrlich selbst von beiden Zellarten entwirft, mußte Zweifel erwecken. Ein durchgreifender Größenuntersehied geht aus ihr nicht hervor, denn wenn Ehrlich auch im allgemeinen die Lymphozyten als kleine, in ihrer Größe den roten Blutkörperchen nahestehende Zellen darstellt, so erkennt er doch bedeutende Größendifferenzen an und erwähnt, daß es im Blute auch normalerweise bei Kindern, insbesondere aber bei Leukämien, ,ganz besonders große Formen (von Lymphozyten)" gibt. In bezug auf die Basophilie des Protoplasma wird auch nur ein quantitativer Unterschied angegeben, indem Lymphozyten als stark, die großen uninukleären Zellen sowie die Übergangsformen als schwach basophil bezeichnet werden. Eine amöboide Beweglichkeit wird der einen wie auch der andern Zellgattung (wie sich später gezeigt hat, mit Unrecht) abgesprochen. Bei der Beschreibung des Kerns fehlt gleichfalls ein durchgreifendes Unterscheidungsmerkmal; zwar wird dem kleineren, runden, stark färbbaren Lymphozytenkern der größere, sçhwächer tingible, ovale oder eingeschnürte Kern der uninukleären Zellen bzw. Übergangsformen gegenübergestellt, gleichzeitig aber auf eine dem $\mathrm{R}$ i e d e $\mathbf{r}$ schen Atlas entnommene

1) Weidenreich, Arch. f. mikr. Anat. Bd. 72, 1908. 
Abbildung verwiesen, welche Lymphozyten mit sehr stark ausgebildeten Einschnürungen und Lappungen der Kerne zeigt.

Selbst Anhänger der Ehrlich schen Dualitätslehre haben Bedenken getragen, sich ihr in der bezeichneten ursprünglichen. Form anzuschließen und es für nötig gefunden, gewisse Änderungen einzuführen. Schon bald darauf wurde

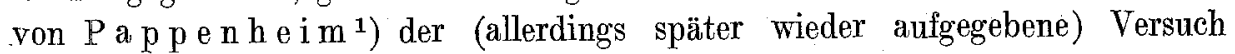
gemacht, die Stellung der Lymphozyten und der großen uninukleären Zellen dahin zu verschieben, daß letztere von den granulierten Zellen abgetrennt und den Lymphozyten angereiht wurden, von $\mathrm{T} \ddot{\mathbf{u}} \mathrm{rk}^{2}$ ) wieder wurden jene Zellen als eine dritte selbständige Gruppe hingestellt, also eine Dreiteilung vorgenommen; wo also der eine eine Lücke in der Formenreihe erblickt, sieht der andere Übergänge und umgekehrt.

Es war natürlich, daß man von der Untersuchung derjenigen Urgane, welche als Bildungsstätten der Leukozyten bekannt waren, Aufschluß in der Streitfrage erwartete, als solche kamen in Betracht $\mathrm{Ly} \mathrm{mphdr}$ ï se $\mathrm{n}, \mathrm{Milz}$ und nach meinen schon im Jahre 1868 gemachten Angaben das Knochen mark. Was man über die zelligen Elemente dieser Organe wußte, schien der Dualitätslehre günstig zu sein, es war bekannt, daß in dieser Beziehung ein Gegensatz bestand zwischen Lymphdrüsen und Milz einerseits, Knochenmark anderseits, insofern das Parenchym der ersteren sich durch seinen Reichtum an kleineren. lymphozytären Zellen auszeichnete, während die „Markzellen" großenteils den größeren granulierten Blutleukozyten so ähnlich waren, daß sie schwer von ihnen zu unterscheiden waren. Die genauere Erforschung der Zellen mit den E h r li c h schen Methoden hatte sodann zur Aufstellung von folgenden zwei Sätzen geführt: 1. Jedes Blutbildungsorgan führtdem Blute Leukozyten bestim m ter Qualität z u. 2. In diesen Organen existieren $\mathrm{z}$ we $\mathrm{i}$ v o $\mathrm{n}$ einander versehiedene selbständige Stammzellen, in den Lymphdrüsen und der Milz eine Stammzelle der Lymphozyten und eine andereim Knochenmarkfür die übrigen farblosen Blutzellen. Diese beiden Behauptungen galten als die wichtigsten Stützen der Dualitätslehre.

Was den e r s t e n Satz betrifft, so läßt sich die Beweiskraft desselben, selbst wenn man seine volle Gültigkeit anerkennt, nicht hoch anschlagen, denn die behauptete Tatsache würde ja auch verständlich sein, wenn die Rollen der Blutbildungsorgane in der Art verteilt wären, dab $n \mathrm{icht}$ jedes derselbe $n$ eine besondere Zellspezies, sondern vielmehr dieselbe Zelle in. versehiedenen Stadien ihrer Entwicklung produzieren w ürde.. Übrigens sind aber die bisher vorliegenden Beobachtungen durchaus nicht geeignet, zuginsten einer solchen durchgreifenden Trennung der Funktionen zu sprechen. Ich kann hierbei nicht unterlassen, nochmals darauf aufmerksam zu machen, wie wenig Sicheres vorläufig

1) P a p pen heim, Virch. Arch. Bd. $159 \mathrm{u}_{\phi}$ 160, 1900.

2) T ïr ks Vorlesungen über klinische Hämatologie. 1904. 
darüber feststeht, in welcher Form die farblosen Blutzellen die blutbildenden Organe verlassen und in den Blutstrom übergehen. Nur für die $\mathrm{L} \mathrm{y} \mathrm{m} \mathrm{ph}$ $\mathrm{drüs}$ en liegen in dieser Beziehung sowohl aus älterer wie aus neuester Zeit (Weidenreich ${ }^{1}$ )) zahlreiche Beobachtungen vor, so daß wir die Elemente der dem Blute zuströmenden Lymphe als genügend bekannt bezeichnen können. Auf das Blut der Milzvenen und der ausführenden Knochenmarksvenen aber haben sich die mit den modernen Hilfsmitteln angestellten Untersuchungen nur selten erstreckt; besonders auffallend ist, daß die Dualisten gerade diese Objekte stark vernachlässigt haben, obwohl dieselben für ihre Argumentationen ohne $Z_{\text {weifel von größter Bedeutung }}$ sind; die von ihnen hauptsächlich kultivierte Methode der Untersuchung eines abgestrichenen Tröpfehens Milz- oder Knochenmarksaftes konnte dafür selbstverständlich keinen genügenden Ersatz liefern und mußte in einseitiger Anwendung zu Irrtümern führen. So erklärt es sich, daß Ehrlich sich sogar über die Vermehrung der Zahl der Leukozyten im Milzvenenblut, welche bereits in früherer Zeit durch Zählungen festgestellt worden ist und auch neuerdings mehrfach bestätigt wurde, hinwegsetzen konnte und auf Grund der schon erwähnten experimentellen, sehr verschiedener Auslegung fähigen Versuche mit Milzexstirpationen mit Bestimmtheit glaubte aušsprechen zu können, daß die Milz keine oder wenigstens eine sehr unbedeutende Rolle als Blutbildungsorgan spiele. Auch bei seinen Anhängern, welche ebenfalls mehr oder weniger dezidiert dieselbe geringe Meinung von der Milzfunlttion äußern, suchen wir vergeblich nach speziellen Untersuchungen über numerische Verhältnisse und Qualität der Leukozyten des Milzvenenblutes; die positive Angabe, daß hier auch größere granulierte und multinukleäre Formen zu finden sind ( Weiden reich), wird übergangen.

Ebenso dürftig und teilweise widersprechend sind die Angaben über das aus dem $\mathrm{Knochenmark}$ der allgemeinen Zirkulation zuströmende Blut. Obwohl ich bereits bei meinen ersten, die myelogene Blutbildung feststellenden Arbeiten in der Erkenntnis der Wichtigkeit einer hierauf gerichteten Untersuchung den Inhalt der Markvenen prïfte und gerade dadurch zu der Überzeugung kam, daß nicht nur, wie bisher allgemein angenommen worden, Milz und Lymphdrüsen, sondern auch das Knochenmark eine Bildungsstätte der farblosen Blutzellen sei, liegen den späteren Untersuchungen über diese Funktion des Knochenmarks fast ausschließlich Beobachtungen über die Elemente des Marksaftes, meist an Trockenpräparaten angestellt, zugrunde, die selbstverständlich über die im Venenblute vorhandenen Zellen keinen Aufschluß geben können. Den Vertretern der Dualitätslehre fehlt daher jede sichere Unterlage für ihre hierauf bezüglichen Behauptungen, die sich vielmehr ihrer Theorie alkkommodieren. Ehrlich selbst hat sich denn auch über diesen Punkt sehr verschieden ausgesprochen; anfänglich ${ }^{2}$ ) vor Aufstellung des Spezifitätsprinzips geneigt, anzunehmen, daß ,die Apparate der Blut-

\footnotetext{
1) Weidenreich, Verhdl. d. Anat. Ges. 1907. - I d em, Die Leukozyten und verwandte Zellformen. 1911, S. 219.

$\left.{ }^{2}\right)$ Ehrli e b, Ztschr. f. klin. Med. I, 1880.
} 
bildung fast nur einkernige Elemente als Rohmaterial in die Blutbahn übertreten lassen und daß diese dann - gleichgültig, ob sie aus Milz, Knochenmark oder Lymphdrüsen stammen - sich in der gleichen einheitlichen Weise verändern", meinte er später ${ }^{1}$ ), daß die durch ihn von den Lymphozyten abgetrennte Form der großen uninukleären Zellen vom Knochenmark aus in die Blutbahn gelange und sich während der Zirkulation in die Form der multinukleären neutrophilen Leukozyten verwandle. Hiermit war es allerdings kaum zu vereinigen, daß seiner gleichzeitigen Angabe nach im normalen Blut ,uninukleäre neutrophile Zellen unter keinen Umständen erscheinen", wie sie doch im Verlaufe der angenommenen Umwandlung auftreten müßten. Anders ist wiederum der in dem $\mathrm{E} \mathrm{h} \mathrm{r} \mathrm{li} \mathbf{c h}-\mathrm{L}$ a $\mathbf{z}$ a $\mathrm{r}$ u s schen Werke ${ }^{2}$ ) vertretene Standpunkt, wonach die multinukleären neutrophilen Leukozyten fertig ausgebildet aus dem Knochenmarksgewebe hervortreten und in das Blut übergehen, während die großen uninukleären Formen und die Übergangsformen nur in geringer $\mathrm{Zahl}^{\mathbf{3}}$ ) diesen Übertritt mitmachen, angeblich weil sie dem chemotaktischen Reize, welcher die multinukleären Zellen hervorlockt, nicht zu folgen imstande sind. Die meisten Vertreter der Dualitätslehre scheinen sich gegenwärtig dem letzteren extremen Standpunkt $\mathrm{Eh} \mathrm{rlichs} \mathrm{angeschlossen} \mathrm{zu}$ haben, ohne daß jedoch zu ersehen wäre, daß sie sich auf spezielle Untersuchungen des Markvenenblutes berufen können und sich nicht mit der allerdings bequemeren Methode der Herstellung von Trockenpräparaten des Marksaftes begnügt haben; es besteht hier eine Lücke im Beobachtungsmaterial, die durch noch so zuversichtliche Aussprüche nicht ausgefüllt werden kann.

Soviel steht aber schon gegenwärtig fest, daß für das Tierreich das $\mathrm{E} \mathrm{hrlich} \mathrm{-}$ $\mathrm{L}$ a $\mathrm{z}$ ar us sche Gesetz nicht gilt; für die Amphibien habe ich bereits im Beginn meiner Untersuchungen über das Knochenmark gezeigt, daß in den Knochenvenen eine so große Anhäufung von kleinen lymphozytären Elementen stattfindet, daß sie nur aus einer starken Einwanderung aus dem umgebenden Gewebe zu erklären ist; für die Vögel liegen die Arbeiten von $\mathrm{D}$ e $\mathrm{n}$ $\mathrm{s}^{4}$ ) und $\mathrm{B}$ i z z $0 \mathrm{z}$ e r $0^{5}$ ), sowie aus der neueren Literatur sehr genaue Untersuchungen von $\mathrm{D}$ a $\mathrm{n} \mathrm{t} \mathrm{s} \mathrm{h}$ a $\mathrm{k}$ of $f^{\mathrm{e}}$ ) und $\mathrm{Ven} \mathrm{s} \mathrm{aff}^{\mathrm{7}}$ ) vor, welche sämtlich darin übereinstimmen, daß die in den Venenanfängen des Knochenmarks auftretenden farblosen Zellen e in $\mathrm{k}$ e r n i g sind; von den letzteren beiden Autoren werden sie demnach auch als Lymphozyten bezeichnet. Thre Abbildungen zeigen keine einzige multinukleäre Zelle; M a x i m ow ferner konstatierte ${ }^{8}$ ) bei kleineren Säugetieren, daß bei der

1) Ehrlich, Charité-Annalen Bd. 12, 1885.

2) Ehrlich-Lazarus, a. a. 0. 1898.

3) A. a. O. S. 74 heißt es sogar, daB, die uninukleären und die Utbergangsformen unter normalen Verhältnissen $\mathrm{nicht}$ in die Blutbahn übertreten", sondern "n u r die reifen granulierten Leukozyten".

4) Denys, La structure de la moelle des os. 1888.

$\left.{ }^{5}\right)$ B i z z o zero, Arch. f. mikr. Anat. Bd. 1890, 25.

$\left.{ }^{6}\right)$ Dantschak of $\mathrm{f}$, ibid. Bd. 74, 1909.

7) Vensl aff, ibid. Bd. 77, 1911.

8) M a xi mow, ibid. Bd. 76, 1910. 
embryonalen Entwicklung des Knoehenmarks zwar Lymphozyten, aber „niemals granulierte Zellen" in die Gefäße eindringen.

Hieran reiht sich die prinzipiell wichtigere Frage, inwieweit es den Vertretern der Spezifitätslehre gelungen ist, den Beweis für ihren $z$ w e it e n Satz, daß in den blutbildenden Organen zwei voneinander verschiedene Stammzellen, ein „Lymphoblast" für die Lymphozyten und ein „Myeloblast“ für die granulierten Zellen, vorhanden sind, zu erbringen. Den ersteren erblicken sie in den sonst als Keimzentrumszellen der Lymphfollikel der Lymphdrüsen und Milz bekannten Elementen, den letzteren in gewissen großen, ungranulierten Zellen des Knochenmarks, von denen es zweifelhaft geblieben ist, ob sie den großen Lymphozyten oder den großen uninukleären Zellen $\mathrm{E} h \mathrm{~h}$ li c h scher Nomenklatur zuzurechnen sind. Die Unitarier bestreiten diese Duplizität der Stammzellen, halten vielmehr daran fest, daß alle Leukozyten aus einem gemeinsamen Stamme hervorgehen, sei es, daß, älteren Anschauungen entsprechend, kleinere Lymphozyten in kontinuierlicher Entwicklung sich zuerst in größere Lymphozyten und später in granulierte Zellen umwandeln, sei es, daß, wie die neueren Erfahrungen es wahrscheinlich gemacht haben, groBe Lymphozyten sich in divergierender Entwicklung einerseits durch Mitosen zu kleinen Lymphozyten, anderseits durch zunehmende Differenzierung zu granulierten Zellen entwickeln. Pap p enheim ${ }^{1}$ ), welcher sich dieser Ansicht in letzter Zeit angeschlossen hat, bezeichnet diese gemeinsame Stammzelle als eine "polyvalente, großlymphozytäre Zelle", "Leukozytoblast" oder ,Myelymphoblast".

Das hier in Betracht kommende Beobachtungsmaterial ist erst kürzlich von Weiden reich in seinem neuen Werke, die Leukozyten und die verwandten Zellformen" zusammengestellt und auf Grund zahlreicher eigener Studien und unter Beibringung neuer Beobachtungen kritisch geprüft worden, es geht daraus hervor, daß die Tatsachen mehr gegen eine Trennung von "Myeloblasten" und "Lymphoblasten" sprechen als dafür, denn es ist unverkennbar, daß die Beobachter bei der Angabe der Unterscheidungszeichen sich untereinander und teilweise mit sich selbst in Widersprüche verwickelt haben. Es sei nur folgendes hierüber angeführt:

Von gewisser Seite ist angegeben worden, daß Nukleolen im Kern nur den Lymphoblasten zukämen, den Myeloblasten dagegen fehlen, spätere Beobachtungen sollten gezeigt haben, daß auch letztere Nukleolen besitzen und zwar in größerer Zahl (2--4), als die Lymphoblasten (1-2). S e h ri d d e findet bei beiden einen oder mehrere Kernkörperchen und bei den Myeloblasten ,eine zarte Membran und ein zierliches Chromatinnetz", bei den Lymphoblasten ,eine dicke Kernmembran" und dunkle, auf Chromatinreichtum beruhende Färbung, Unterschiede, welche jedenfalls auf seiner der Abhandlung im Ztlbl. f. Path. 1908, Nr. 24 beigegebenen Abbildung sehr wenig deutlich hervortreten. Gewicht ist besonders auf den Umstand gelegt worden, daß nach einer modifizierten A I t m a n $\mathrm{n}$ schen Färbungsmethode in den sonst homogen erscheinenden Lymphoblasten gewisse

i) Pappenheim, Folia haematol. 1908. 
azidophile Granulationen sichtbar zu machen sind, in den Myeloblasten dagegen nicht, jedoch wurde bereits von $\mathrm{Fr}$ e if eld angegeben, daß bei einer andern Modifikation jener Methode auch in den Myeloblasten sich azidophile Granula erkennen lassen, die sich von jenen der Lymphoblasten nur durch einige Besonderheiten unterscheiden lassen; indessen ist von $\mathrm{Mewe}^{1}$ ) der Nachweis geführt worden, daß die Altmann-Schriddeschen Granula nichts anderes sind als die schon früher von Benda ${ }^{2}$ ) bei den multinukleären Leukozyten des Menschen und von ihm selbst an den Leukozyten des Salamanderblutes dargestellten, dem Protoplasma als ,genuine Bestandteile" zukommenden Chondriosomen, seine Abbildungen zeigen sie in gleicher Weise bei Lymphozyten und multinukleären Zellen, also bei Abkömmlingen b e i d e r Stammzellen; auch ist durch die Angaben anderer neuerer Autoren ( $\mathrm{Butterfield,} \mathrm{Heineke} \mathrm{und} \mathrm{Meyer,}$ St. K le in) der Befund der S e h ridde-Alt man n schen Granula als Unterscheidungsmerkmal zwischen Lymphoblasten und Myeloblasten entwertet worden. Ebenso haben die zuerst von $\mathrm{Mi} \mathrm{ch}$ a elis und $\mathrm{W}$ olf f erwähnten azurophilen Granulationen, welche mit Hilfe der R o m a n $0 \mathrm{w}$ s $\mathrm{k}$ y schen Färbung zum Vorschein kommen, die ihnen zugewiesene Bedeutung als Erkennungsmittel der Lymphoblasten genaueren Prüfungen gegenüber nicht behaupten können, G r a wit z fand sie auch in „Myeloblasten“ und ist geneigt, sie für eine Vorstufe der neutrophilen Granulationen zu halten. - Daß es sich bei der Basophilie des Protoplasmas höchstens nur um quantitative und daher bedeutungslose Verschiedenheiten handelt, ist wohl allgemein anerkannt, $\mathrm{S} \mathrm{c} \mathrm{hridde} \mathrm{nennt} \mathrm{beiderlei} \mathrm{Zellen}$ „mittelbasophil“, und was schließlich die von $\mathrm{Brandenburg}$ eingeführte Oxydasereaktion des Protoplasmas (Blaufärbung durch Guajak) betrifft, die von einzelnen Autoren als ,gegenwärtig bei der Differentialdiagnose zwischen lymphatischen Elementen und Zellen der Leukozytenreihe ausschlaggebend" angepriesen worden ist, so hat sich auch die auf sie gesetzte Hoffnung als trügerisch erwiesen, da $\mathrm{St}$. K le i n auch bei Lymphozyten, die sich gegen dieselbe refraktär verhalten sollten, einen positiven Ausfall konstatierte; ihre Unzuverlässigkeit ging übrigens auch bereits aus W. Sehultzes Angabe ${ }^{3}$ ) hervor, ,daß die normalen Myeloblasten des Knochenmarks die Reaktion für gewöhnlich nicht geben, wohl aber die pathologiseh im Blut und in den Organen vorkommenden Myeloblasten bei der Myeloblastenleukämie g r ö B t e n t e il s "; also weder bei positivem noeh bei negativem Ausfall läßt sich von ihr eine Entscheidung erwarten.

Wenn aus dem Gesagten schon hervorgeht, in wie unvollkommener Weise von seiten der Dualisten bisher der Aufgabe, charakteristische Eigentümlichkeiten der von ihnen angenommenen beiden Stammzellen anzugeben, genügt ist, so erseheint noch eine aus der Durchsicht ihrer Abhandlungen, soweit sie mir zugängig waren, sich ergebende Betrachtung bemerkenswert: da Lymphozyten und granulierte Zellen sich fast stets, wie von diesen Autoren neuerdings zugegeben wird, in den

1) Me we s, Arch. f. mikr. Anat. Bd. 75, 1910.

a) B e n d a, Verh. d. Phys. Ges. Berlin 1899.

3) W. Schultze, Münch. med. Wschr. 1909 Nr. 4. 
blutbildenden Organen vereint zeigen, und da ferner anzwnehmen ist, daß beiderlei Zellen keine stabilen, sondern vielmehr beständig sich erneuernde Elemete sind, so ließe sich, wenn sie verschiedenen Stammes wären, erwarten, daß auch häufig $\mathrm{n}$ e b e n e in a n d e $\mathrm{r}$ in demselben Objekt, also z. B. in dem abgestrichenen Knochenmarksaft, g 1 e i c h $\mathrm{z}$ e i ti g Lymphoblasten und Myeloblasten sich zeigen müßten; nirgends aber wird ein solcher Befund erwähnt, alle in Frage kommenden Zellen werden e $\mathrm{n}$ twe der als Lymphoblasten oder als Myeloblasten aufgefaßt; sie in ein und demselben Präparat zu unterscheiden und in zwei Kategorien $z \mathfrak{u}$ sondern, scheint den Beobachtern nie gelungen und ihnen auch nicht in den Sinn gekommen zu sein, obwohl hier gerade ein Prüfstein für ihre Lehre ihnen in die Hand gegeben war.

Eine interessante Illustration des vergeblichen Bemühens, zwei verschiedene Stammzellen der Lymphozyten und der Myelozyten auseinanderzuhalten, liefert ferner auch eine auf dem später ausführlicher zu behandelnden pathologischen Gebiete sich abspielende Diskussion, nämlich der Streit darüber, wohin die bei der durch Ebstein bekannt gewordenen akuten Leukämie im Blute auftretenden farblosen Zellen gehören. Nachdem bereits Askanazy ${ }^{1}$ ) hier in einem Falle als vorherrsehendes Element große, uninukleäre, großenteils granulafreie, zum Teil aber auch neutrophil granulierte Zellen beschrieben hatte, wurde dieser Befund bald darauf von Fränke ${ }^{2}$ ) als ein bei akuter Leukämie konstanter festgestellt, wenn auch die von $\mathrm{A}$ s k a n a $\mathrm{z}$ y erwähnte neutrophile Körnung eines Teiles der Zellen meistens vermißt wurde. Auch Ehrlich und Lazarus sowie Pincus (a.a. O. S. 18) finden, daß bei akuten Leukämien ,die kleinen Lymphozyten an Häufigkeit meistens zurücktreten und das Bild von den großen Lymphozyten beherrscht wird". Die hier gewählte Bezeichnung der Zellen als ,große Lymphozyten“, welcher sich auch bereits Fränkel bedient hatte, wurde sodann trotz des Widerspruches von

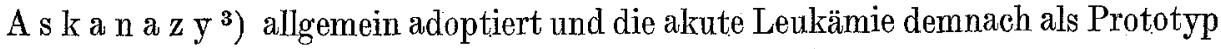
einer lymphatischen Leukämie hingestellt, dieselbe somit als ein Erzeugnis der Erkrankung des ,Iymphoiden Apparates“, und zwar (wegen der öfters nur unbedeutenden Anschwellungen der Milz und der Lymphdrüsen) vorzugsweise des im Knochenmark enthaltenen lymphoiden Gewebes betrachtet. Die Größe der Zellen, ihre Granulationslosigkeit und die Beschaffenheit führten auch zu einem Vergleich mit den Stammzellen der Lymphozyten, den Lymphoblasten und zu der Bezeichnung der akuten Leukämie als „Lymphoblastenleukämie“. Diese Einigkeit wurde gestört, als W. Schultz $\mathrm{e}^{4}$ ) durch seine Beobachtungen $\mathrm{zu}$ dem Resultate geführt wurde, daß von den akuten Lymphoblastenleukämien gewisse Fälle abzusondern seien als ,akute Myeloblastenleukämien“; er gesteht zwar ein, daß die Unterschiede in morphologischer Beziehung sehr gering seien, ,die Größe

1) M. A s ka nazy, Virch. Arch. Bd. 137, 1894.

2) A. Fränkel, D. med. Wsehr. 1895.

$\left.{ }^{3}\right)$ M. A s kanazy, D. med. Wschr. 1895 .

$\left.{ }^{4}\right)$ W. S c h u l t z e, Zieglers Beitr. Bd. 39, 1906. - I d e m, Münch. med. Wschr. 1909, Nr. 4, 
beider Zellen ist fast gleich, das Protoplasma in beiden basophil, die äußere Konfiguration des Kerns genau übereinstimmend, Untersehiede bestehen nur in der feineren Kernstruktur und darin, daß die Lymphoblasten einen schmalen, hellen Hof um den Kern besitzen". S chultzes Hauptargument war der positive Ausfall der schon besprochenen Oxydasereaktion und das Verhalten der Milz und Lymphdrüsen, in welchen nicht lymphatische, sondern „,myeloblastische Elemente" gewuchert waren, eine Angabe, welche natürlich nur dann zu verwerten wäre, wenn die Spezifität dieser myeloblastischen Elemente seibst feststände und nicht erst zu beweisen wäre; da dies nicht der Fall ist, bewegt sich die Deduktion in einem Circulus vitiosus.

In S e hultzes Fußtapfen sind dann aber trotzdem alsbald andere Untersucher getreten ( Pappenheim, K. Ziegler, Voswinkel und Danzelt), sie bestätigen das Vorkommen einer Myeloblastenleukämie, geben aber keine unterscheidenden Merkmale an, sondern berufen sich wiederum hauptsächlich auf die „myeloiden Metaplasien“ der blutbildenden Organe, die sie konstatier̨t zu haben glauben, von der vorgefaßten Meinung ausgehend, daß die „myeloiden“ Zellen dieser Organe spezifisch verschieden von den Lymphozyten und Lymphoblasten sind. Übrigens heben diese Vertreter der Doppelnatur der akuten Leukämie hervor, daß wahrscheinlich manche früher als Lymphoblasten geltende Befunde irrtümlich gedeutet worden seien und es sich in Wirklichkeit wohl um Myeloblasten gehandelt hätte, ein Beweis mehr, wie groß die Ähnlichkeit ist, wenn ein solcher Verdacht aufkommen kann, und daß die Vermutung, es beständen vielleicht überhaupt keine Unterschiede, durchaus berechtigt jst.

Eine besondere Erwähnung verdienen an dieser Stelle noch die B e $\mathrm{n} d$ a schen Angaben. Dieser Autor hat für die Stammzellen der Lymphozyten (Keimzentrumszellen) den Namen , L y m p h o g o n i e n " und für die Stamm(Keim-)formen des Markelements den Namen "M yelog o nien" vorgeschlagen; er gibt die ,außerordentliche Ähnlichkeit" beider zu, erkennt aber doch gewisse Unterscheidungszeichen an mit der Reservation, daß er ,bezweifeln müsse, daß die Lymphogonien in jedem Falle von den gleichfalls ins Blut gelangenden Myelogonien zu unterscheiden seien". In bezug hierauf haben nun Ehrlich und Pincus selbst an der letztbezeichneten Stelle ihrer Arbeit auffälligerweise die Bemerkung gemacht, daß ,kein zwingender Grund" für die Annahme vorliege, daß die B e n da schen Lymphogonien und Myelogonien untersehieden werden müßten; damit werden aber auch zugleich Untersehiede zwischen Lymphoblasten und Myeloblasten, geleugnet, denn es liegt doch auf der Hand, daß diese letzteren Ausdrücke Synonyma sind und sich mit den B e n d a sehen Bezeichnungen decken.

Es fehlt übrigens auch sonst nicht an Äußerungen von seiten der Ehrlich schen Schule, in welchen ein Eingeständnis liegt, daß sie die Schwierigkeit, ihre Stellungnahme aufrecht zu erhalten, erkennt. So finden wir bei W.Schult ze (l. c.) den Ausspruch: „Die Unterschiede sind so gering, daß nur erfahrene Histologen, wie Schridde, mit Sicherheit Myeloblasten und Lymphoblasten unterscheiden kön- 
nen"; andere frühere Anhänger der Dualitätslehre, welche sich mit einer solchen, ëinem Autor zugeschriebenen persönlichen Verantwortlichkeit nicht begnügen wollten, haben sich der Lehre abgewandt: „Ich erkenne keine morphologisehen Unterschiede prinzipieller Natur zwischen Lymphoblasten und Myeloblasten an, doch soll das mögliche Vorhandensein solcher nicht geleugnet $\operatorname{sein}^{\prime \prime}$ (Pappen heim). Der einzige Ausweg, welcher sich den Dualisten in diesem Dilemma bietet, scheint mir in einer allerdings nicht direkt von der Hand zu weisenden Hypothese zu liegen, es käme nämlich in Frage, ob nicht trotz aller morphologischen, und wie es nach den Reaktionen scheint, auch chemischen Übereinstimmung zwischen Lymphoblasten und Myeloblasten ein Unterschied zwischen beiden durch ihre $\mathrm{En} t$ w i c $\mathrm{kl} \mathrm{u} \mathrm{ng}$ s fähigkeit nach verschiedenen Richtungen erwiesen sei, wie etwa zwei anscheinend ganz gleich beschaffene Ovula ihre Verschiedenheit dadurch kundgeben, daß sie sich zu zwei ganz verschiedenen Organismen entwickeln. Dieser Gedanke scheint eine gewisse Berechtigung zu haben, doch werden wir in Erwägung zu ziehen haben, daß, wenn zwei Zellen einen verschiedenen Entwicklungsgang einschlagen, hieraus allein nicht ihre innere, virtuelle Verschiedenheit folgt, es muß sich auch nachweisen lassen, daß diese verschiedene Entwicklung eine k onstante Erscheinung ist, daß sie unter allen Unständen, bei allem Wechsel der äußeren Einflüsse unabänderlich vermöge der den Zellen innewohnenden, ihnen gewissermaßen angeborenen Kräfte erfolgt, ebenso wie ein $\mathrm{Ei}$, wenn es überhaupt zur Entwicklung gelangt, immer nur einen ganz bestimmten Organismus aus sich entstehen läßt und zu einer Entwicklung nach einer andern Richtung ganz unfähig ist. Für die Stammzellen der Dualisten läßt sich dieser Nachweis nicht führen, da wir ihre Entwicklung unter verschiedenen Bedingungen, an den mannigfach wechselnden Orten, an welche sie ihre Wanderungen im Körper führen, nur sehr unvollkommen zu verfolgen vermögen. Wer wollte z. B. die Möglichkeit in Abrede stellen, daß ein aus dem Keimzentrum einer Lymphdrüse, also von einem Lymphoblasten stammender Lymphozyt, welcher daselbst nur neue Generationen von Lymphozyten produziert hätte, durch die ausführenden Lymphgefäßße in das Blut gelangt und in das Knochenmark eingeführt, hier als Myeloblast uns entgegentritt und zur Mutterzelle granulierter Leukozyten wird? Diese Möglichkeit müssen auch die Dualisten gelten lassen, wenn sie das erwähnte Zugeständnis machen, daß Lymphoblasten und Myeloblasten morphologisch und chemisch sich nicht unterscheiden lassen.

Wir kommen somit zu dem Schlusse, daß es einstweilen an Beweisen dafür fehlt, daß die in dem Blute, der Lymphe und den Blutbildungsorganen vorkommenden verschiedenen Formen farbloser Blutzellen in zwei voneinander verschiedene, selbständige Gruppen zerfallen; sie bängen vielmehr wahrscheinlich sämtlich durch eine gemeinsame, auch im postembryonalen Leben stets vorkommende großlymphozytäre Stammzelle untereinander zusammen. In welcher Weise sich diese Stammzelle immer wieder ergänzt, ob ausschließlich durch mitotische Teilung oder auch aus andern Zellen, insbesondere aus den fixen Zellen der mesenchymatösen Gebilde, 
kann hier unerörtert bleiben; es sei nur bemerkt, daß letztere Ansicht durch die neuen embryologischen Untersuchungen von $\mathrm{Maximow}{ }^{1}$ ) und $\mathrm{Dantscha \text {- }}$ $k \circ f^{2}$ ) eine wichtige Unterstützung erfahren hat. Für eine Beteiligung des Gefäßendothels während des extrauterinen Lebens fehlen sichere Anhaltspunkte. - Eine weitere, sich unmittelbar ergebende Schlußfolgerung aus der gegebenen Darstellung wäre die, daß aus dieser gemeinschaftlichen Stammzelle, welche in allen blutbildenden Organen vorhanden ist, sich innerhalb letzterer auch immer alle Arten von Leukozyten bilden können und daß daher auch im Blutstrome nicht aus der Beschaffenheit der einzelnen Zellen mit Bestimmtheit auf ihren Ursprung zu schließen ist.

Nach der gegebenen Darstellung dürfte es wünschenswert sein, die von der $\mathrm{Eh}$ r I i c h schen Schule eingeführte Terminologie in einigen Punkten abzuändern. Den in letzter Zeit mehrfach angefeindeten Ausdruek "L y mphozyte.n " werden wir, dà er eine im allgemeinen gut abgegrenzte Gruppe $\mathrm{k} l \mathrm{e}$ in e $\mathrm{r}$ Zellen umfaßt, schon mit Rücksicht auf eine alte Tradition beibehalten können, wenn er auch seine ursprüngliche, auf den alleinigen Ursprung aus den Lymphdrüsen hinweisende Bedeutung nicht mehr in Anspruch nehmen kann. Den Lymphozyten gegenüber wären alsdann alle granulierten Leukozyten zu stellen, sei es als Leukozyten sensu strictiori oder als Granulozyten oder, was vielleicht am meisten sich empfiehlt, als ,, M y e l o z y t e n ", da diese Zellen ja hauptsächlich, wenn auch nicht ausschiließlich, im Knochenmark vertreten sind, ebenso wie die Lymphozyten in den Lymphdrüsen; eine Beschränkung des Begriffs der My elozyten auf die einkernigen granulierten Zellen, wie sie gegenwärtig gebräuchlịch ist, kann füglich fortf a $1 l$ e $\mathrm{n}$ und die Unterscheidung zwisehen ein- und mehrkernigen granulierten Zellen sowie die Bezeichnung der verschiedenartigen Granulationen einem adjektivischen Zusatz überlassen bleiben. Die als „Lymphoblasten“ und „Myeloblasten“ bezeichnete Elemente aber müssen unter einen gemeinschaftlichen Namen zusammengefaßt werden, und da sie vermutlich mit den $\mathrm{Eh} \mathrm{r} \mathrm{li} \mathrm{chschen,} \mathrm{g} \mathrm{r} \mathrm{o} B$ e $\mathrm{n}$ Lymphozyten" zu identifizieren sind, so erscheint dieser Name für sie anwendbar, wenn man nicht den etwas unbequemeren Ausdruck „Lymphomyeloblast". oder "Lympholeukoblast" vorzieht.

Wie bekannt, hat die Ehrli ch sche Spezifitätslehre in pathologischen Beobachtungen, insbesondere in den Befunden bei der L e u kämie, eine Stütze zu finden geglaubt, ja man kann sagen, daß sie auf dem Boden dieser Beobachtungen entstanden ist. Nachdem wir ihre Berechtigung auf Grund der Resultate der normalhistologischen Forschung in Zweifel gezogen haben, erwächst uns die Aufgabe, zu zeigen, daß auch die pathologischen Zustände des Blutes keinen genügenden Beweis für ihre Richtigkeit liefern können. Es handelt sich dabei vor allem

1) M a ximow, Arch. f. mikr. Anat. Bd. 73, 1909.

2) Dantschak of $f$ ibid. Bd. 73, 74. 1909, 1910. 
um die Auffassung der Beziehungen der Le u kämie zu den Blut bild ung s or ga ne n.

Daß solche Beziehungen bestehen, erscheint selbstverständlich, und fast allgemein anerkannt ist auch, daß die Erkrankung dieser Organe der leukämischen Blutbeschaffenheit zugrunde liegt, daß letztere also eine sekundäre Folge der ersteren ist und nicht eine primäre selbständige Erkrankung des Blutes darstellt. Seitdem Vir ch ow diesen Standpunkt eingenommen hatte, haben alle späteren Versuche, hieran zu rütteln, nichts zu ändern vermocht, und auch Loew it 's sehr beachtenswerte Arbeiten, welche für eine parasitäre primäre Bluterkrankung eintreten, haben nicht diese Ansicht erschüttern können. Ich habe die Gründe, welche für die Blutbildungsorgane als Ausgangspunkt der Erkrankung sprechen, schon früher an anderem Orte $^{1}$ ) angeführt und hebe hier nur als wesentliches Moment hervor, daß Erkrankungen dieser Organe konstant sind und daß sichere Fälle von Leukämie ohne dieselben nicht bekannt sind, wie zu erwarten wäre, wenn sie sich primär entwickelte, Seitdem meine Untersuchungen gelehrt haben, daß auch das Knochenmark zu diesen Organen gehört, stehen auch die vereinzelten Fälle, in denen Milz und Lymphdrüsen frei von Erkrankung zu sein schienen, nicht mehr in Widerspruch zu obigem Satze, er hat vielmehr durch den Nachweis einer Erkrankung des Markes in solchen Fällen eine neue Bestätigung erfahren. Beobachtungen, in welchen auch im Knochenmark keine pathologische Veränderung gefunden werden konnten, sind immer auf berechtigte Zweifel gestoßen und sind in der so umfangreichen Leukämieliteratur älterer und neuerer Zeit auch nur in äußerst geringer Zahl zu finden. Es läßt sich vermuten, daß, wie im folgenden näher begründet werden soll, sie sich teils aus Mangelhaftigkeit der auf die Untersuchung verwandten Sorgfalt, teils an den geringen Fortschritten, welche der pathologische Prozeß noch zur Zeit der Sektion gemacht hatte, erklären lassen; die Anfänge einer Erkrankung können sicher leicht übersehen werden. - Was übrigens die Natur der in den Blutbildungsorganen sich abspielenden pathologischen Vorgänge betrifft, so wird an dem h y p e $\mathrm{p} l$ as $\mathrm{t}$ i s c h e n Char a k t e r derselben festzuhalten sein. Es handelt sich ohne Zweifel um aktive, proliferative Prozesse, wobei die Parenchymzellen des hämatopoetischen Systems eine abundante Vermehrung, wie die zahlreichen Mitosen zeigen, erfahren, Milz und Lymphdrüsen bedeutend an Volumen zunehmen und auch das Mark auf Kosten der umgebenden Knochensubstanz sich, wie ich schon in meinen ersten Beobachtungen konstatieren konnte, erheblich expandieren kann; die Zellen aber, welche durch diesen hyperplastischen Prozeß erzeugt werden, zeigen meistens denselben Charakter, wie die Zellen des Blutes, und daher geht der ly mphozytären, groBlymphoz ytären und myelozytären Leukämie ${ }^{2}$ ) parallel eine lympho-

1) E. N e u m a n n, Berl. klin. Wschr. 1878, Nr. 6, 7, 9, 10.

2) Teh folge in der Bezeichnung großenteils den Vorschlägen von W al z (a. a. O.). - Die von M in k ow ski (Verhdl. des Kongr. f. inn. Med. 1899) entworfenen klinischen Krankheitsbilder der genuinen Leukämie, der akuten L. und der chronischen Lymphämie entsprechen, wie es scheint, ziemlich vollständig obiger anatomischen Einteilung. 
zytäre,groBlymphozytäre und myelozytäre Hyperplasie des. Knoehenmarks, der Milz und der Lymphdrüsen.

Die Rückwirkung dieser Organerkrankungen auf das Blut wird nun von der hèrschenden Lehre so formuliert: Eine ,myeloide“, d. h. myelozytäre Leukämie, ausgezeichnet durch den Reichtum des Blutes an größeren granulierten Zellen, entsteht bei Wucherung des myeloiden Gewebes, d. h. des Markes inkl. des myeloiden Apparates der andern Organe, dagegen bildet sich eine lymphatische, i. e. lymphozytäre Leukämie mit starker Vermehrung der Lymphozyten bei Wucherung der Lymphdrüsen inkl. des sonstigen lymphatischen Apparates, die Untersuchung des Blutes würde also über den Ursprung entscheiden. Meine Beobachtungen hatten mich dagegen, da sie mir zeigten, $d a ß$ bei der Leukämie in ein und demselben Organ Zellen verschiedener Art, granulierte Zellen und Lymphozyten entstehen können, dahin geführt, den Satz aufzustellen, daß die Untersuchung des Blutes zwar die Beschaffenheit der durch denWucherungsprozeß in den Blutbildungsorganen erzeugten Zellen anzeigt, aber keinen Rückschluß auf den Sitz dieser Wucherung in einem bestimmten Organ gestatte. „Die Qualität der farblosen Zellen im leukämischen Blute ist hauptsächlich abhängig von dem Charakter, den der hyperplastische Proze $\beta$ in dem erkrankten Organ annimmt oder, genauer gesagt, von der Beschaffenheit der von ihm daselbst produzierten Zellen, nicht aber von dem Ursprungsort." Hervorzuheben ist, daß diese Divergenz der Ansichten weniger aus einer Verschiedenheit der Beobachtungstatsachen als vielmehr auf der verschiedenen Beurteilung allseitig anerkannter anatomischer Befunde entsprungen ist und daß die verschiedene Deutung auf die eben besprochene Differenz der Auffassungen des Verhältnisses der farblosen Blutzellen zu einander zurückzuführen ist.

Ich habe meine Ansicht hauptsächlich begründet auf die von mir am le $\mathrm{uk}$ ämis chen $\mathrm{Kn}$ och e $\mathrm{n} \mathrm{m}$ ar $\mathrm{k}$ gemachten Beobachtungen.

Der zweite von mir im Jahre 1872 beschriebene Fall leukämischer Knochenmarkserkrankung betraf einen Fall von V i r c how scher Lymphämie bei einem 53jährigen Manne, und hier wurde von mir zum ersten Male eine lymph ozy täre H yperplasie (Iymphoide Metaplasie) des Markes festgestellt, Milz und. Lymphdrüsen waren gleichzeitig stark geschwellt; das Knochenmark war in ein makroskopiseh und mikroskopisch den lymphdrüsen ähnliches Gewebe umgewandelt, und seine Wucherung hatte sehr deutliche Arrosionen der Knochensubstanz herbeigeführt; seine zelligen Elemente bestanden fast ausschließlich aus ,kleinen freien oder von sehr schmaler Protoplasmalage umschlossenen Kernen“" und „nur vereinzelten ausgebildeten Zellen und großen runden Mutterzellen, welche eine größere Zahl jener kleinen Lymphkörperchen entbielten“. „Diese kleinen lymphoiden. Elemente lagen dichtgedrängt in den Maschen eines ziemlich dichten, jedoch sehr unregelmäBigen Retikulum"; ausdrücklich erwähnt ist der Unterschied der Zellen gegenüber den im normalen Mark vorkommenden ,größeren, wohlausgebildeten, kernhaltigen Zellen“, die ich früher bereits im Falle meiner ersten Beobachtung leukämischer Knochenmarksaffektion (1870) ausführlicher beschrieben hatte. In Milz und Lymphdrüsen stimmten die eingelagerten „Lymphkörperchen" ebenfalls ,,mit den im Blute so zahlreich vertretenen Gebilden überein“, außerdem fanden sich auch hier öfters ,große, zahlreiche kleine Lymphkörperchen umschlieBende Zellen" "1).

1) Das Vorkommen dieser eigentümlich en, den äIteren Beschreibungen von „Mutterzellen mit endogener Zellbrut" entsprechenden Elemente in leukämischen Hyperplasien und Neubildungen ist später ganz in Vergessenheit geraten. 
Seitdem weiß man, daß eine solche lymphoide Umwandlung des Markes, besser als lymphozytäre Hyperplasie bezeichnet, einen regelmäßigen Obduktionsbefund der lymphozytären (sogenannten ,lymphatischen“) Leukämie bildet, wenn man von den wenigen zweifelhaft gebliebenen Fällen absieht, in welchen eine Markaffektion überhaupt gefehlt haben soll.

Das ist eine Tatsache, über deren Deutung zwisehen Dualisten und Unitariern hauptsächlich gestritten wird. Ersteren hat die Erklärung Schwierigkeiten bereitet, da sie nicht zugeben konnten, daß aus den normalen „myeloiden Elementen“" des Knochenmarks lymphoide Zellen hervorgehen, sie wurden von ihnen daher anfänglich als aus den Lymphdrüsen und dem sonstigen lymphatischen Apparate, dem angeblich alleinigen Ausgangspunkte der lymphatisehen Leukämie, in das Knochenmark eingeführte Fremdlinge betrachtet; E h r l i c h - L a z a r u s (a. a.O. S. 115) rangieren demnach den Befund in eine Linie mit andern ,leukämischen Metastasen", z. B. in der Leber. Die Unhaltbarkeit dieser Erklärung ergibt sich schon mit großer Wahrscheinlichkeit aus dem diffusen, nicht herdweisen Auftreten der Veränderung und aus ihrer allgemeinen Verbreitung über das ganze Skelett, sie wird ferner erwiesen durch die Feststellung, daß es Fälle lymphatischer Leukämie gibt, in welchen der lymphatische Apparat völlig intakt oder wenigstens nur in geringfügiger Weise affiziert war, worauf ich später zurüekkomme. Es muß daher auffallen, daß noch in neuester Zeit einzelne Autoren an dem Gedanken einer Metastase im Knochenmark festhalten, so sagt $\mathrm{K}$. Z i e g l e ${ }^{1}{ }^{1}$ : „ „Für die lymphatische Umwandlung des Knochenmarks ist meiner Ansicht nach kaum eine andere Erklärung möglich als die durch Kolonisation von aus den Blutgefäßen stammenden Zellen“, und doch gibt derselbe Autor gleichzeitig zu, daß ,es Fälle lymphatischer Leukämie mit lymphatischer Umwandlung des Knochenmarks o h n e Vergrößerung der Milz und der Lymphdrüsen gibt" !

Die vorherrschende Ansicht der Dualisten ist gegenwärtig jedenfalls eine andere, man rekurriert auf das im Knochenmark selbst, wenn auch nur in geringer Menge vorhandene „lymphatische Gewebe“, welches bisher unberücksichtigt geblieben war. Ehrli ch selbst hat sehr bald sich dieser Auffassung zugewandt, denn sehon in dem zweiten, die Leukämie speziell behandelnden, von ihm in Gemeinschaft mit Pin c u s bearbeiteten Teile seines Werkes wird die schon lange bekannte Existenz lympoider Zellen im Knochenmark anerkannt, dieselben als Bestandteile eines ,lymphatischen Gewebes" dargestellt und von ihnen die lymphoide Wucherung des Markes abgeleitet, letzterem allerdings nur eine untergeordnete Stelle neben der als Hauptherd der lymphatischen Leukämie hingestellten Hyperplasie der Lymphdrüsen und übrigen lymphatischen Apparate zugewiesen, ,im Gegensatze zu der Genese der myeloid leukämischen Metastasen bildet sich der lymphatisch leukämische Tumor an Ort und Stelle an jedem beliebigen Punkte des Körpers aus den daselbst normal bereits vorhandenen Spuren lymphatischen Gewebes" (a. a. O. S. 70). „Die Veränderungen des Markes bestehen wie die der übrigen Organe in einer alleinigen Wucherung der Lympho-

1) K. Zi e gle r, Ztschr. f. klin. Med. Bd. 72, 1911. 
z y te $n$, die in dem beschränkten Raume des Markkanales a 11 es. a ndere ruhende Gewebe verdrängt und ersetzt ${ }^{1}$ ). Das funktionierende Parenchym des Knochenmarks bleibt bis auf die mechanische Verdrängung unbeteiligt an dem ganzen Prozeß so wie in jedem andern Organe, z. B. in Leber und Niere, die Parenchymzellen unbeteiligt bleiben" (a. a. O. S. 30). - In gleichem Sinne haben Schridde u. a. von einer Erdrückung des ,m yeloischen Parenchyms" dureh das wuchernde lymphatische Gewebe gesprochen.

Nach diesen mit großer Sicherheit hingestellten Behauptungen müßte man erwarten, daß ihnen genaue histologische Untersuchungen des lymphoiden Knochenmarks zugrunde liegen und nicht etwa bloß eine Abstraktion aus der Theorie, nach welcher myeloide und lymphoide Zellen etwa so wenig ineinander übergehen können wie Nervenzellen und Bindegewebszellen. An solchen Untersuchungen fehlt es aber fast gänzlich; in dem zitierten Ehrli ch schen Werke wird meine sehr unvollkommene, zur Beantwortung der Fragen, um die es sich hier handelt, jedenfalls ganz ungenügende Beschreibung des lymphoid umgewandelten Knochenmarks reproduziert, ergänzende Angaben über die Gewebsstruktur haben nur wenige Forscher geliefert; fast ausschlieBlich beschränken sich die Untersuchungen auf Beschreibungen der verschiedenen Zellen, wie sie sich an Trockenpräparaten unter Anwendung mannigfaltig modifizierter Färbungsmethoden darstellen, aber auch hier wird wenig auf den Kardinalpunkt, nämlich auf die Entstehung der Zellen, eingegangen; einen Beweis dafür, daß nur die Lymphoidzellen wuchern und dab die ,Myeloidzellen" nicht an der Wucherung teilnehmen, wie es doch behauptet wird, vermißt man, eine regressive Metamorphose und Atrophie der letzteren, wie sie etwa bei den durch lymphatische Wucherungen erdrüekten Leberzellen eintritt, ist nirgends beschrieben. Den Grund für diese Lücken der Untersuchung kann keinesfalls darin gesucht werden, daß zur Zeit der Sektion der Prozeß immer bereits abgelaufen ist und die Umwandlung beendet ist, denn bekanntlich verlaufen ja gerade die lymphozytären, namentlich die großlymphozytären (lymphatischen) Leukämien öfters sehr akut, und die Veränderungen im Knochenmark erscheinen an der Leiche nicht sehr vorgeschritten, es müßte also zu konstatieren sein, daß nur die lymphoiden Zellen Mitosen zeigen, die myeloiden Zellen nicht, es müßte sich zeigen, daß neben den in großer Zahl vorhandenen „Lymphoblasten“ auch die dem normalen Mark zukommenden „Myeloblasten“ in gewisser Zahl zu finden sind. Nur durch diese Angaben könnte die Ansicht der Dualisten über. den Prozeß erwiesen werden.

So lange dies nicht der Fall ist, wird die gegenteilige Auffassung der Vertreter der unitarischen Lehre ebenfalls ihre Berechtigung behalten, welche dahin geht, daß a 11 e Zellen des Knochenmarks an der hyperplastisehen Wucherung teilnehmen, ebensowohl die Lymphozyten als die größeren granulierten Zellen, insbesondere aber die gemeinschaftlichen Stammzellen beider, die großen Lymphozyten (oder Lymphomyeloblasten); das pathologische Moment würde, abgesehen von der starken

1) Im Text nicht gesperrt! 
Proliferation, darin zu suchen sein, daß die junge Zellbrut die Fähigkeit verliert, sich in normaler Weise zu Myelozyten zu entwickeln und daher in mehr rudimentärem Zustande der Lymphozyten verharrt. Es muß zugestanden werden, daß die Ursachen, welche diese Produktion unvollkommen ausgebildeter Zellen bedingen, vorläufig nicht angegeben werden können, aber ebensowenig können ja die Dualisten Rechenschaft darüber ablegen, weshalb, wie sie annehmen, nur die eine Zellgruppe wuchert, die andere nicht.

Der eben besprochenen lymphoiden Umwandlung des Knochenmarks bei lymphatischer Leukämie stellt sich als ein für die Theorie der Leukämie gleichfalls wichtiger Befund, die myelozytäre Hyperplasie (sogenannte "myeloide Metaplasie") der Milzund Lymphdrüsen, bei der "Myelämie" Ehrli chs zur Seite. Es handelt sich dabei um die erst durch neuere Beobachtungen bekannt gewordene Tatsache, daß auch im Gewebe dieser Organe in Fällen starker Vermehrung der großen granulierten farblosen Blutzellen im Blute mehr oder weniger reichlich diese Zellen auftreten, obwohl sie im normalen Zustande der Hauptsache nach nur kleinere lymphoide Zellen enthalten. Der Besprechung dieses Gegenstandes seien einige Worte über die m y e l o z y tär e Hy p e r plasi e des Knochenmarks selbst vorausgeschickt.

Daß in diesen Fällen sogenannter myeloider, myeloischer oder myelozytärer Leukämie das Knochenmark den Ausgangspunkt der Erkrankung bildet und daß die Blutzellen also Knochenmarkszellen sind, steht seit meiner ersten Publikation, in welcher ich mich für den myelogenen Ursprung aussprach, fest. Demnach ist auch kein Zweifel darüber, daß die Veränderungen des Markes, von neueren, vor Paradoxen nicht zurückschreckenden Autoren „,myeloide Umwandlung“ genannt, autochthoner Natur sind und sich an den daselbst präexistierenden Zellen abspielen. Aber trotzdem besteht wieder eine prinzipielle Differenz über ihre Entstehung; der dualistischen Auffassung, daß die Myelozyten und polymorphkernigen granulierten Markzellen allein und zwar auf Kosten der Lymphoidzellen wuchern, stellt sich die Ansicht der Unitarier, nach der beiderlei Zellen bei dem Proliferationsprozeß beteiligt sind, und beide gemeinsam Zellprodukte erzeugen, welche den Charakter der ersteren Art an sich tragen, gegenüber. Leider sind auch hier die Untersuchungen noch so wenig vorgeschritten, daß eine definitive Entscheidung zugunsten der einen oder der andern Ansicht einstweilen nicht getroffen werden kann. Die feineren Gewebsveränderungen bei dem makroskopisch doch höchst auffällig sich bemerkbar machenden pathologisehen Prozesse, welcher in ausgebildeten Fällen das Knochenmark in eine eiterklumpenähnliche Masse umwandelt, so daß ich früher die Bezeichnung ", p y o ide H y perpla s i e " gebrauchte, sind noch heutigen Tages so gut wie unbekannt, und die auf die Untersuchung der morphologisehen Bestandteile des Knochenmarksaftes beschränkte Untersuchung hat sich als unzureichend erwiesen; als Hauptbefund wird stets die große Zahl der "Myeloblasten“ angegeben, wir haben aber gesehen, daß eine Unterscheidung dieser Zellen von den Lymphoblasten nicht möglich ist und demnach auch eine Beteiligung derlymphozytären Zellen des Knochenmarks an der Proliferation nicht auszuschließen ist. 
Was nun die namentlich durch die Arbeiten $\mathrm{Hirs} \mathrm{chfelds}$ bekannt gewordenen myelozytären Hyperplasien (,myeloiden Metaplasien") der Milz und Lymphdrüsen, welche die besprochene „pyoide" oder "myelozytäre“" Hyperplasie des Knochenmarks öfters begleiten, betrifft, so haben wir bei der Deutung dieser interessanten Erscheinung von der gegenwärtig wohl allgemein anerkannten Tatsache auszugehen, daß, wie bereits oben erwähnt, auch normal große uninukleäre und multinukleäre granulierte Zellen dem Milz- und Lymphdrüsengewebe keineswegs fremd sind und daß es sich in dem bezeichneten pathologischen Zustande lediglich um eine mehr oder weniger bedeutende Zunahme derselben handelt. Über die physiologische Lokalisation der fraglichen Zellen in den genannten Organen sind unsere Kenntnisse übrigens noch lückenhaft. F le m m in g fand sie in den Keimzentren normaler Lymphdrüsen und überhaupt in den Knoten und Strängen adenoider Substanz nur vereinzelt liegen, konstatierte aber ibr massenhaftes Vorkommen im Gewebe der Drüsenkapseln und der Trabekel; in der Milz ist den neueren Angaben von Weide n reich und Dominici zufolge in der roten Pulpa, namentlich in der Umgebung der Follikel, ihr Hauptherd zu suchen, letztere jedoch enthalten ebenfalls eine gewisse Zahl derselben. Mit diesen Angaben steht die pathologische Erfahrung bei der "myeloiden Metaplasie" im Einklang; es scheint nämlich festzustehen, daß bisweilen bei derselben die Myelozyten sich ebenfalls mit Vorliebe in bestimmter Weise lokalisieren, in der Milz in der roten Pulpa, in den Lymphdrüsen zwischen Follikeln und Marksträngen, also in dem lockeren Gewebe der Lymphsinus und in den bindegewebigen Trabekeln, während das adenoide Gewebe der Follikel selbst in beiden Organen sich frei von jenen Zellen erhält und daher ein Follikelschwund durch den Druck des umgebenden großzellig infiltrierten Gewebes zustande kommt. Daß diese Beobachtungen allgemein gültig sind, muß freilich bezweifelt werden; ein Überblick über die Literatur läßt vermuten, daß es jedenfalls nicht als ausnahmslose Regel hingestellt werden kann, daß bei der Myelozytämie die Vergrößerung der Milz mit einer Atrophie der Follikel und im Gegensatz hierzu bei der Lymphozytämie mit einer Anschwellung derselben auf Kosten der Pulpa verbunden ist; selbst in der Beschreibung, welche Ehrlich - Pincus (a. a. O.) von der Milz bei beiden Formen der Leukämie geben, ist ein solches gegensätzliches Verhalten der Follikel und der Pulpa nicht

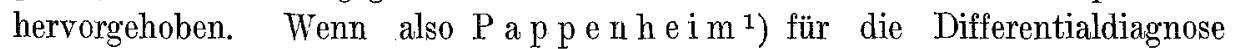
,einen sicheren Ausweis darin erblickt, daß es bei myeloblastischer Leukämie zu einer myeloiden Pulpametaplasie in der Milz und zu myeloider interfollikulärer Hyperplasie der Drüsen kommt", zum Unterschiede von der Lokalisation der lymphatischen Leukämie, so vermißt man genauere Angaben über das Beobachtungsmaterial, auf welches er sich stützt. Auf einem Mißverständnis kann es aber nur beruhen, wenn ein neuerer Autor auch einen Gegensatz zwischen dem Verhalten der Follikel einerseits und der Markstränge in den Lymphdrüsen anderseits konstruiert, indem er sagt: „Die myeloide Umwandlung beginnt stets in den Marksträngen, nie in

1) Pappenheim, Fol. haematol. Bd. 5, 1908.

Virchows Archiv f. pathol. Anat. Bd. 207. Heft 3. 
den Follikeln, diese zeigen alle Grade der Atrophie" (K. Zi.egler, a. a. O.).

Für die Deutung der myeloiden Metaplasie der Milz und Lymphdrüsen kommen wieder dieselben Fragen in Betracht, welche bei der Besprechung der lymphoiden Metaplasie des Knochenmarks uns entgegentraten: E h r li c h, welcher die Präexistenz myelozytärer Elemente dort verkannte und das Knochenmark allein als normalen 'Träger des „Myeloidgewebes" anerkannte, trat anfänglich für eine Entstehung durch "Me tastase“ von letzterem Organe aus ein: „in Milz und Lymphdrüsen nisten sich nach Art von Metastasen Herde von Myeloidgewebe ein“" (Ehrlich-L a zarus, a. a. O. S. 116), „daß die sekundären leulkämischen Tumoren in inneren Organen bei der myelogenen Leukämie metastatisch entstehen durch Verpflanzing des fremden Markgewebes aus dem Knochenmark hat große Wahrscheinlichkeit für sich" ( $\mathrm{Ehrlich-Pincus,} \mathrm{a.} \mathrm{a.} \mathrm{O.} \mathrm{S.} \mathrm{70).}$ Nachdem nun die Voraussetzung bezüglich des normalen histologischen Baues von Milz und Lymphdrüsen, aus welcher diese Lehre hervorging, sich als irrig erwiesen hat, ist sie nicht mehr aufrecht zu erhalten, insbesondere wenn berücksichtigt wird, wie wenig Ähnlichkeit das Verhalten leukämisch geschwellter Milzen und Lymphdrïsen mit dem bekannten Bilde metastatischer Tumoren bositzt. Auch Anhänger der dualistischen Auffassung der Blutleukozyten haben Widerspruch dagegen erhoben und erklärten ,die Lehre von der Kolonisation für irrig“" (Schridde). So sind wir denn, ebcuso wie für die lymphozytäre Hyperplasie (lymphoide Metaplasie) des Knochenmarks bei der Lymphämie, auch hier wieder darauf angewiesen, auf pathologische Vorgänge an den a u to hth o nen Elementen der Milz und der Lymphdrüsen bei diesem Zustande zurückzugreifen. Daß über die Art und den Angriffspunikt des pathologischen Erozesses durch die bisherige, fast ausschließlich kultivierte Methode der Untersuchung eine genügende Aufklärung herbeigeführt worden ist, wird niemand behaupten wollen; der Beeinflussung der Beurteilung des Zustandes durch eine vorgefaßte Theorie ist immer noch ein weiter Spielraum gelassen.

Von seiten der Dualisten wird die Vermehrung der Myelozyten auf eine Neubildung derselben aus ihren Stammzellen, den „Myeloblasten“, zurückgeführt, während vom unitarischen Standpunkte aus auch den Iymphozytären Zellen eine Beteiligung an ihrer Produktion zugeschrieben wird. Den Beweis dafür, daß nur die ,Myeloblasten“" in Betracht kommen, mußten jene jedenfalls schuldig bleiben, da, wie wir gesehen haben, sie selbst zu dem Eingeständnis gelangt sind, dab „Myeloblasten " und ,Lymphoblasten" morphologisch und chemisch in ihren Eigenschaften übereinstimmen, sie auch niemals den Versuch gemacht haben, in Präparaten von leukämischen Milzen und Lymphdrüsen diese beiden von ihnen angenommenen Stammzellen auseinanderzuhalten. Es liegt daher sehr nahe, zu vermuten, daß wenigstens ein Teil der von ihnen gefundenen ,Myeloblasten“ aus den Lymphozyten hervorgegangen war und daß auch letztere zu dem Reichtum an Myelozyten beigesteuert haben. Die vorhin erwähnte, öfters von neueren Autoren beschriebene vorzugsweise Anhäufung der Myelozyten in der roten Milzpulpa und 
in dem intérfodikulären Gewebe der Lymphdrüsen kann deshalb nicht für ihren Ursprung aus präformierten Zellen derselben Art angefübrt werden, weil auch an den genannten Orten inmer neben den Myelozyten Lymphozyten vorkommen, letztere sogar in überwiegender Zahl. Daß aber die Follikel beider Organe, wie angegeben wird, bei dem Prozeß bisweilen erdrückt werden und atrophieren, würde sich aus einer starken expansiven Wucherung des sie umgebenden Gewebes erklären lassen; gleichviel, ob diese Wucherung nur die Myelozyten oder gleiehzeitig auch die Lymphozyten des letzteren betrifft. ${ }^{1}$ ) Nichts scheint also der Berechtigung der Annahme, daß die Eigentümlichkeit der bei der myelozytären Leukämie in der Milz und den Lymphdrüsen einșetzenden Wucherung darin besteht, daß die durch sie erzeugten jungen Zelldegenerationen, unabhängig von ihrer Herkunft, die Neigung haben, sich zu größeren granulierten Zellen, zu Myjelozyten, zu entwickeln und daß die Lymphozytenproduktion dagegen eingeschränkt oder sistiert wird, entgegenzustehen.

Vor einem ,non liquet" stehen die Unitarier freilich wiederum, wenn sie sagen sollen, wodurch diese Tendenz zu einer höheren Entwicklung der jungen Zellen bedingt ist, welche dem Wucherungsprozeß der lymphatischen Leukämie fehlt; dieser Mangel ist aber nicht größer als die Ratlosigkeit der Dualísten gegenüber der Frage, weshalb bei der einen Form der Leukämie nur das „myeloide Gewebe", bei der andern nur das lymphatische Gewebe in Wucherung gerät.

Etwas anders als bei der myelozytären Hyperplasie der Milz und Lymphdrüsen liegen die Verhältnisse bei der Bildung der sekundären, myelozytären Infiltrationen und Tumoren im Gefolge der Myelämie in dem Bindegewebe anderer Organé, Leber, Niere usw. Ein „Myeloidgewebe" als normaler Bestandteil ist bisher außerhalb der blutbildenden Gewebe noch nicht entdeckt worden, wenn man nicht etwa im Bindegewebe überall gelegentlich vorkommende Wanderzellen oder emigrierte, granulierte, polymorphkernige Blutleukozyten dafür gelten lassen will. Es dürfte daher hier nur die Alternative in Betracht kommen: Emigration der Zellen aus dem myelämischen Blut, also eine Art metastatischer Vorgang, oder autochthone Entstehung aus lymphozytären, vielleicht den fixen Bindegewebszellen entstammenden Elementen. Die Schwierigkeit, eine Entscheidung über diese beiden Annahmen zu treffen, ist genugsam aus den umfangreichen, trotz des überreichen, nach allen Richtungen hin durchgearbeiteten, großenteils experimentellen Beobachtungsmaterials immer noch nicht zum Abschluß gebrachten Diskussionen über die Herkunft der bei entzündlichen Prozessen auftretenden mannigfaltigen Zellformationen bekannt, und ich will diese Frage nicht aufs neue aufrollen. Es sei nur hervorgehoben, daß, wenn die Dualisten sich in Konsequenz ihres Prinzips für die Emigrations-(oder Metastasen-)hypothese unter Zuhilfenahme unbekannter chemotaktischer Kräfte, welche die Zellen herauslocken, ențcheiden müssen, damit für

1) Wie sich leicht ergibt, sind aus dem angegebenen Grunde auch die Schlußfolgerungen, welche aus der bei lymphatischer Leukämie beobachteten Follikelvergrößerung auf Kosten der Milzpulpa und dem entsprechenden Verhalten der Lymphdrüsen zugunsten der Spezifizitätslehre abgeleitet hat, nicht stichhaltig. 
síe ein neues Dilemma entsteht; denn es erseheint kaum wahrscheinlich, daß ein durch Emigration entstandener Krankheitsherd später die Zelleñ wieder durch Immigration an das Blut abgibt und zu einer neuen Quelle für die Zunahme der Leukozyten im Blute wird, wie gerade von seiten der Dualisten angenommen zu werden scheint; beide einander entgegengesetzten Vorgänge scheinen sich vielmehr gegenseitig auszuschließen.

Auch sind die Vertreter der metastatischen Entstehungsweise der myelämischen sekundären Erkrankungen beliebiger Organe gezwungen, dieselben in bezug auf ihre Genese gegenüberzustellen den bei Lymphämie so häufig multipel auftretenden Bildungen lymphatischen Gewebes im Bindegewebe versehiedener Organe, denn für diese gilt ihnen ja der autochthone Ursprung als feststehend: „Der lymphatisch-leukämische Tumor entsteht (im Gegensatze zu den myeloiden leukämischen Metastasen) ganz wie das entzündliche Lymphozyteninfiltrat, an Ort und Stelle an jeder beliebigen Stelle des Körpers aus den dort bereits normal vorhandenen Spuren lymphatischen Gewebes, alle Gewebe werden hierdurch von einem lymphadenoiden Netze durchzogen, in welches - gegen die Norm an Zahl und an Größe vermehrt - bedeutendere Lymphome eingelagert sind" ( $\mathrm{Ehrlich-P} \mathrm{i} \mathrm{n} \mathrm{c} \mathrm{u} \mathrm{s} \mathrm{,}$ a. a. O. S. 70). Die unitarische Lehre, welche eine solche Gegenüberstellung der sekundären Affektionen bei Lymphozytenlenkämie und Myelozytenleukämie nicht anerkennt, nimmt auch für ihre Entstehung bei letzterer jene ubiquitär verbreiteten „Spuren lymphatischen Gewebes" in Anspruch und erhebt gegen die Trennung den Finwand, daß die bei der Wucherung letzterer entstehenden „Lymphoblasten“" sich, wie erörtert, nicht von den „Myeloblasten" des Knochenmarks unterscheiden lassen, also auch kein Grund vorliegt, ihnen die gleiche Fähigkeit, Myelozyten zu erzeugen, abzusprechen. Die verschiedene Beschaffenheit der entstehenden Neubildungen wäre alsdann dadurch zu erklären, daß bei der Lymphämie die wuchernden Zellen ebenso wie bei der sich hier entwickelnden lymphozytären Hyperplasie des Knochenmarks, der Milz und der Lymphdrüsen nicht zu der höheren Ausbildung gelangen wie bei der Myelämie, was auf eine Verschiedenheit der einwirkenden unbekannten Noxen zurückgeführt werden kann. Selbstverständlich werden von diesem Gesichtspunkt aus betrachtet in jedem Falle von Leukämie, gleichviel welcher Qualität die farblosen Blutzellen sein mögen, die hyperplastischen Erkrankungen der blutbildenden Organe und die Neubildungen außerhalb derselben als his tog e netis ch k o ordiniert gelten müssen, und die Bezeichnung „sekundär" für die letzteren würde nur in dem Sinne zu gebrauchen sein, als sie wohl stets zeitlic h der Affektion der Blutbildungsorgane nachfolgen.

Nach dem Vorausstehenden werden wir die Frage, ob sich eine Einteilung der Leukämien in eine,,myeloide" und eine „lymphatische": Form im Sinne Ehrlichs aufrecht erhalten läßt, verneinen müssen. Diese Einteilung gründet sich auf die Annahme, daß es zwei voneinander ganz verschiedene Systeme von Zellen und Geweben gibt und daß bei der Leukämie entweder das eine oder das andere System in hyperplastische Wucherung gerät, eine Annahme, welche sich vorläufig nicht erweisen läßt. Eine Unterscheidung nach der Lokalisation in einem bestimmten 
Leukozytensystem läßt sich nicht durchführen. Ebensowenig aber können wir der Ansicht beitreten, då $B$ sich aus der Blutbeschaffenheit ein Rücksehluß auf ein bestimmtes Organ machen läßt, welches der Sitz der hyperplastischen Wucherung ist. Sowohl das Knochenmark als die Milz und die Lymphdrüsen enthalten nebeneinander, wenn auch in sehr ungleichem Verhältnis, Lymphozyten und Myelozyten, jedes dieser Organe kann bei der Leukämie eine gesteigerte Menge von Zellen beiderlei Art dem Blute zuführen, der ursprüngliche Typus der proliferierenden Zellen braucht sich dabei nicht zu erhalten, wenigstens sprechen die Beobachtungen keineswegs dagegen, daß Myelozyten von Lymphozyten und diese von jenen produziert werden, entgegen dem Spezifitätsgesetz. Der von mir früher ausgesprochene Satz, daß aus der Blutuntersuchung zwar der Charakter des Prozesses, d.h. die Beschaffenheit seiner Produkte, abernicht der Ursprungsort erkannt werden könne, dürfte auch gegenwärtig zu Recht bestehen gegenüber der Behauptung von Ehrlich und Lazarus (a. a. O. S. 119): „Die mikroskopische Blutuntersuchung am gefärbten Trockenpräparat allein, ohne Zuhilfenahme irgendwelcher andern klinischen Methode, ist jmstande, zu entscheiden, ob eine Leukämie vorhanden ist und ob dieselbe der lymphatischen oder der myelogenen Form angehört."

Utber die Beteiligung der einzelnen Blutbildungsorgane an der Leukämie kann nur die anatomische Untersuchung Aufsehlub g e b e n, und was hierüber bekannt geworden, hatte mich vor fast 35 Jahren $^{1}$ ) dahin geführt, zu behaupten, daß es Fälle rein myelogener Leukämie mit alleiniger Erkrankung des Knochenmarks gäbe und daß kein Fall beobachtet wäre, in welchem das Knochenmark normal gefunden wäre und die Leukämie lediglich von der Milz und den Lymphdrüsen hergeleitet werden könnte; daraus wurde von mir die Schlußiolgerung gezogen, daß wahrscheinlich jede Leukämie myelogenen Ursprungs sei.

Überblickt man die neuere Literatur, so ergibt sich, daß die Bedeutung des Knochenmark als Sitz der primären, in einzelnen Fällen der einzigen Erkrankung für die $\mathrm{M} \mathrm{yeloz} \mathrm{y} \mathrm{a} \mathrm{mi}$ e allgemein anerkannt ist und pathologischen Befunden in Milz und Lymphdrüsen nur eine nebensächliche Bedeutung zugeschrieben wird. Zweifelhaft ist es nur geblieben, welche Rolle dem Knochenmark bei der L y $\mathrm{m}$ p h 0 z y tä mi e zukommt, doch auch hier wird die Konstanz seiner Erkrankung in Verbindung mit der von Lymphdrüsen und Milz fast von allen Autoren zugegeben. Der Berechtigung, auch für den letzteren Fall einen myelogenen Ursprung der Leukämie anzunehmen, wird entgegengestellt 1 . daß es Fälle gibt, in welchen die Lymphdrüsen und Milz in viel vorgeschrittenerem Grade affiziert waren als das Knochenmark, 2. daß in Fällen, wo die Erkrankung der Lymphdrüsen und der Milz in ihrer Intensität zurücktrat gegen diejenige des Knochenmarks oder wohl auch gänzlich zu fehlen schien, eine Rückbildung jener stattgefunden haben konnte, 3. daß von einzelnen Autoren das Knochenmark bei der Untersuchung

1) E. N e u m a n n, Berl. klin. Wschr. 1878 a. a. O. u. 1880, Nr. 20. 
als: normal angegeben worden ist. Diesen Einwänden gegenüber mag folgendes bemerkt sein:

Es läßt sich erwarten, daß die Intensität der Knochenmarkserkrankung im allgemeinen entspricht der Zeitdauer ihres Bestehens, letztere läßt sich aber nicht aus dem mehr oder weniger akuten oder chronischen Verlaufe der Krankheit erkennen, da das Knochenmark erst längere Zeit, nachdem andere Symptome bereits sich gezeigt hatten, erkrankt und damit eine leukämische Blutbeschaffenheit als ein neues Symptom herbeigeführt worden sein konnte, oder mit andern Worten: die Schwellungen der Lymphdrüsen und der Milz können sich früher entwickelt haben und deshalb stärker ausgebildet sein als die Hyperplasie des Knochenmarks, und letzteres dennoch der Ausgangspunkt für die Leukämie sein. Es gibt ja sicher konstatierte Fälle, in denen eine Lymphämie erst auftrat, nachdem längere Zeit hindurch eine typische „Psendoleukämie" mit starken Drüsentumoren und Milzschwellung bestanden hatte und die von mir früher für diese Beobachtungen gegebene Erklärung, daß eine sekundär hinzugetretene Erkrankung im Knochenmark diesen Wechsel bedingt, dürfte mehr Wahrscheinlichkeit für sich haben als die Annahme, daß von den erkrankten Lymphdrüsen erst nach langem Bestande eine auffällige Einwirkung auf das Blut ausgegangen ist. In Übereinstimmung hiermit spricht sich E. Grawitz ${ }^{1}$ ) dahin aus, daß,,bei der Pseudoleukämie das Knochenmark nicht primär ergriffen ist, sondern nur in seltenen Fällen sekundär affiziert wird, ein Ereignis, das gleichbedeutend mit dem Übergang in Leukämie sein dürfte". Für diese Auffassung spricht es, daß die Sektionsbefunde unkomplizierter Pseudoleukämie bisher niemals einen der leukämischen „,lymphoiden Metaplasie“ des Knochenmarks gleichenden Zustand dargeboten zu haben scheinen, wohl aber diejenigen, welche Fälle mit einem leukämischen Endstadium betrafen; hiergegen kann auch die Angabe von Ehrlich-Pincus (a.a.O. S. 88), daß das Knochenmark bei der Pseudoleukämie ,häufig und in genau derselben Weise verändert gefunden wird wie bei der ausgesprochenen lymphatischen Leukämie", nicht geltend gemacht werden, da sie ganz allgemein gehalten ist und keinen Unterschied macht zwischen einfachen und mit Leukämie komplizierten Pseudoleukämien. Die klinische Feststellung einer Koinzidenz des Beginns der Vermehrung der farblosen Blutzellen mit der ersten Entwicklung des pathologischen Prozesses im Knochenmark wird freilich immer unmöglich bleiben, da letztere sich der Diagnose am Krankenbett entzieht.

Der $\mathrm{zw}$ e ite Einwand richtet sich gegen die Beweiskraft der Beobachtungen, in welchen das Zustandekommen einer Lymphämie im Gefolge einer Markaffektion ohne Beteiligung der andern Blutbildungsorgane von mir angenommen worden ist. Daß auch bei dieser Form der Leukämie Fälle vorkommen, in welchen weder die klinische Beobachtung noch die Autopsie eine Erkrankung der Lymphdrüsen und der Milz erkennen läßt, dagegen ein entschieden pathologisch verändertes Mark sich zeigt, wird freilich auch kaum mehr in Zweifel gezogen, wenigstens wird dies für die akute lymphatische Leukämie von Ehrlich-

1) E. Grawitz a. a. 0. p. 475 . 
Pin ou sugegeben, sie sagen (a. a. O. S. 60): „Die Tatsache des Vorkommens a us s chlie $B$ li c h e ${ }^{1}$ ) Knochenaffektion trifft vor allem für die akute Leukämie zu", und sie tragen nur deshalb Bedenken, hieraus einen Beweis für myelogenen Ursprung zu entnehmen, weil , v e r m u $t$ l i $\mathrm{c} \mathrm{h}^{1}$ ) die übrigen lymphatischen Apparate gleichfalls an der Hyperplasie beteiligt und nur durch die rapide Ausfuhr ihrer Produkte in die Blutbahn hinein jede klinisch und anatomisch nachweisbare Vergrößerung der Lymphdrüsen, der Milz und anderer lymphatischer Organe verhindert wird" (a. a. O. S. 10); aus dieser Begründung erhellt wohl hinreichend, daß die an andern Stellen (S. 31, 71) von denselben Autoren positiv behauptete „hochgradige Zellproduktion" auch in den nicht vergrößerten Lymphdrüsen und andern lymphatischen Apparaten von ihnen nicht als sicher erwiesen angesehen wird. Hierauf deutet auch hin, daß sie noch zu einer andern Vermutung ihre Zuflucht nehmen, daß nämlich eine lymphoide Hyperplasie der Drüsen zwar den Beobachtern wegen der Gleichheit der Zellen im normalen und pathologischen Zustande leicht entgehen konnte, die lymphoide Erkrankung des Knochenmarks dagegen einen viel auffälligeren Befund wegen der von der Norm abweichenden: Beschaffenheit der Zellen darbot und deshalb allein die Aufmerksamkeit auf sich gezogen hätte (a. a. O. S. 61). Daß ein solcher lrrtum bisweilen vorgekommen ist, läßt sich allerdings nicht widerlegen, jedenfalls aber wird man schwerlich in Abrede -stellen können, daß selbst, wenn der mikroskopische Nachweis einer Beteiligung der übrigen Organe an dem Prozeß in solchen Fällen geführt würde, doch das Hauptmoment in der Erkrankung des Knochenmarks gesucht werden müsse.

Von ausschlaggebender Bedeutung für die Entscheidung über die von mir angenommene myelogene Natur aller Leukämien ist natürlich der d ritte, dagegen erhobene Einwand, sich beziehend auf die Angaben einiger Autoren, daß sie das Knochenmark intakt gefunden hätten. Auf eine Kritik eines jeden einzelnen dieser Fälle einzugehen, ist sehon deshalb nicht möglich, weil nur eine Nachprüfung derselben ein sicheres Urteil gestatten würde; ich muß mich darauf beschränken, hervorzuheben, daß die Anfangsstadien der Erkrankung des Knocbenmarks nur durch eine genaue mikroskopische Untersuchung festgestellt werden können, namentlich gilt dies für die akute Leukämie, welcher mehrere der in Frage stehenden Fälle angehören. Ehrlich-P in eu s haben selbst sich dahin geäußert, daß ,die Angaben über das Mark in diesen anscheinend negativen Fällen nicht den Anforderungen der modernen Untersuchungstechnik entsprechen und daß bei gellauerer Prüfung sich wohl gewisse Abweichungen von der Norm ergeben haben würden" (a. a. O. S. 61), und an anderer Stelle (a. a. O. S. 10) heißt es: „Die gewöhnlich als Beweisstück für die Leukämie ohne anatomisches Substrat zitierten Fälle von $\mathrm{Leube}$ und Fं eischer, Henek bieten völlig ausreichende Veränderungen des Markes dar." Makroskopisch kann sich die Erkrankung sicher leicht der Beobachtung entziehen, da weder eine Farben- noch eine Konsistenzveränderung notwendig vorhanden zu sein braucht, mikroskopisch ist bei Lymphozytämie als positiver Befund nur eine mehr oder weniger beträchtliche Vermehrung

1) Im Original $n \mathrm{i}$ e h t gesperrt! 
der lymphoiden Zellen im Marksaft zu erwarten, während bei Myelozytämie wegen der Homologie der durch den hyperplastischen Prozeß neugebildeten Zellen eine Veränderung ihrer Qualität nicht zu konstatieren ist. Um so größeren Wert können zwei negative Merkmale beanspruchen, die trotz meines und später auch A s $\mathrm{k}$ a $\mathrm{n}$ a $\mathrm{zys}$ Hinweis keine genügende Beachtung gefunden haben, wir sind nämlich berechtigt, jedes rote Mark (vom Fettmark natürlich abgesehen) als pathologisch anzusehen, in welchem kernhaltige rote Blutzellen fehlen und aus welchem sich post mortem nichtdie Chareotschen Kristalle a uscheiden, denn beide Erscheinungen sind, wie ich gezeigt habe, in jedem normalen roten Marke zu finden ${ }^{1}$ ). Bei der Leukämie scheint eine Verminderung der Zahl der kernhaltigen roten Blutzellen im Marke sehr gewöhnlich stattzufinden, nicht selten ist ihre Bildung, namentlich bei der Lymphozytämie, ganz sistiert; noch häufiger ist bei dieser Form der Leukämie infolge des veränderten Zellmaterials (Schwund der eosinophilen Zellen ?) ein Ausbleiben der Kristallabscheidung, während die Myelozytämie dieselbe bekanntlich, wahrscheinlich infolge einer starken Anhäufung eosinophiler Zellen, sehr begünstigt. Von seiten Ehrlichs und seiner Schule sind diese Tatsachen übergangen, und doch läßt sich nicht bezweifeln, daß, ebenso wie das Auftreten kernhaltiger roter Blutzellen und kristallinischer Abscheidungen im leukämischen Blute ein klinisehwichtiges Zeichen füreineausdem Knochenmarkabstammende Leakämieist, ebenso ihr Fehlenan der Leiche im Mark unfehlbar anzeigt, dab dieses $\mathrm{krank}$ is t ${ }^{2}$ ).

Für die Begründung der Behauptung, daß in einem Falle von Leukämie das Mark intakt gefunden worden sei, wird also auch eine Angabe darüber erforderlich sein, wie dasselbe sich bei einer auf diese Punkte gerichteten Untersuchung verhalten habe; diesem Erfordernis entspricht, soweit mir bekannt, keiner der Fälle ${ }^{3}$ ), in denen jene Behauptung aufgestellt worden, wohl aber sind Beobachtungen über Leukämiefälle mehrfach publiziert worden, in denen bei Beachtung der erwähnten Befunde ein normal erseheinendes Mark als pathologisch sich erwies;

1) E. N e u mann, Ztlbl. f. d. med. Wiss., 1868, Nr. 44 u. 1869, Nr.19. - Virch. Arch. Bd. 116, 1889. - M. A skan a zy, ibid. Bd. 137, 1894.

2) Bei der meistens akut verlaufenden großlymphozytären Leukämie fehlen $\mathrm{Ch}$ a r c ot sche Kristalle, wie es scheint, in der Regel im Knochenmark und im Blut; daß sie aber auch hier vorkömmen können, lehren Beobachtungen von M. Askanazy, Fraenkel und Benda ; solche Leukämien nehmen also auch in dieser Beziehung eine Mittelstellung zwischen lymphozytären und myelozytären Leukämien ein. - Beiläufig sei hier auch eines andern, makroskopisch verwertbaren von mir angegebenen Unterschiedes zwischen den verschiedenen Formen der Leukämie gedacht, den jeh nirgends erwähnt finde. Blutgerinnsel mit Anhäufungen myelozytärer Elemente erscheinen eitergelb, Gerinnsel mit Lymphozytenanhäufungen $\mathrm{milchweiß.}$

s) In der Beobachtung von $\mathrm{L}$ e $\mathrm{u}$ be und $\mathrm{Fl}$ e is $\mathrm{e} \mathrm{h}$ er wird zwar ausdrücklich das Fe h l e $\mathrm{n}$ Ch a r o ot scher Kristalle erwähnt, aber nicht als Kennzeichen einer lymphoiden Markmetaplasie gewürdigt und das Mark trotzdem als normal bezeichnet. Virch. Arch. Bd. 83, 1881. 
es sei hier nur ein Fall von akuter Lymphozytämie von A s k a n a z y zitiert, in welchem ,das makroskopisch kaum kenntlich veränderte Mark erst durch das fast völlige Fehlen junger roter Blutzellen sich als hochgradig alteriert verriet". Ebenso teilt $\mathrm{D}$ e $\mathrm{n} \mathbf{n} \mathrm{ig}$ einen von W a l z untersuchten ähnlichen Fall mit, in welchem das Mark der Rippen und des Sternum embryonale rote Blutzellen vermissen ließ, aber makroskopisch ganz normal sich verhielt. So unvollständige Beschreibungen, wie in dem viel zitierten Fle is cher-Pen z old schen Falle: „Das Mark verhielt sich der Farbe, Konsistenz und dem mikroskopischen Aussehen nach vollkommen normal" können wirklich nicht genügen, besonders da Sternalschmerzen bei dem Kranken aufgetreten waren; daß mit keinem Worte des Verhaltens der kernhaltigen roten Blutzellen gedacht wird, erklärt sich wohl nur dadurch, daß die Beobachtung in eine Zeit fällt, wo ihre konstante Anwesenheit im roten Mark noch nicht überall anerkannt war. Daß aber auch neuere Beobachtungen, die in gleichem Sinne verwertet sind, hinreichende Zuverlässigkeit nicht besitzen, geht aus dem Gesagten hervor, und ich glaube, daß es, um z. B. die Beobachtung von $H$ irs o h l a f f, in welcher bekanntlich alle wahrnehmbaren Veränderungen der blutbildenden Organe zu fehlen schienen, zu erklären, der von $\mathrm{E} \mathrm{h} \mathrm{r} \mathrm{li} \mathrm{c} \mathrm{h-P} \mathrm{i} \mathrm{n} \mathrm{c} \mathrm{u} \mathrm{s}$ (a. a. O. S. 10) ausgesprochenen Hypothese kaum bedarf, daß ,eine rapide Ausschwemmung nirgends, auch im Knochenmark nicht, eine stärkere Anhäufung lymphatischen Gewebes zustande kommen ließ" ${ }^{1}$ ). - Es sei hier bemerkt, daß über die normale Beschaffenheit des Markes in den einzelnen Knochen des Skeletts, insbesondere über die normale Verbreitung des physiologisch wichtigen roten Markes, immer noch zum Teil sehr irrtümliche Vorstellungen zu bestehen scheinen, obwohl ich vor längerer Zeit ${ }^{2}$ ) auf Grund ausgedehnter systematischer Untersuchungen ein hierfür im allgemeinen gültiges Gesetz aufgefunden habe, nach welchem das rote Mark in den spongiösen Knochen des Stammes von der Geburt an persistiert, in den Extremitätenknochen jedoch, wo es anfänglich gleichfalls vorhanden ist, sich in zentripetaler Richtung von den Phalangen bis zum Humerusund Schenkelkopf in Fettmark umbildet, um sich bei der zur Blutregeneration führenden UmwäIzung umgekehrt in zentrifugaler Richtung wieder zu rotem Mark zurückzuverwandeln. Es muß hiernach höchst auffällig erscheinen, wenn ein Beobachter wie E. Grawitz sagt: ,Man muß sich von der Vorstellung freimachen, daß nur die Röhrenknochen als Matrix der roten Blutzellen anzusehen seien" (a. a. O. S. 98).

Wir sind hiermit dahin gelangt, die Ansicht vertreten zu müssen, daß für einen Teil der leukämischen Erkrankungen der myelogene Ursprung gesichert, für einen andern Teil wegen der wahrscheinlich konstanten Mitbeteiligung des Marks an der pathologisch-anatomischen Veränderung der Milz und der Lyinphdrüsen wenigstens sehr wahrscheinlich ist, wir haben die Möglichkeit nicht ausschließen können, daß in der letzteren Reihe von Fällen Milz und Lymphdrüsen

1) Vgl. die zutreffende Beurteilung der Fälle von Hirschlaff und Koermoeczy bei Walz a. a. O. 1901.

2) E. N e u.m a n n, Ztlbl. İ. d. med. Wiss. 1882, Nr. 18. 
bisweilen den primären Ausgangspunkt der Frkrankung bilden und daß demnach auch neben einer myelogenen Leukämie eine solche lienalen oder lymphatischen Ursprungs vorkommt. Es scheint sogar zugunsten dieser Annahme angeführt werden zu können, daß, wenn - wie wohl kaum bestritten wird - bei der Leukämie Milz und Lymphdrüsen durch ihre pathologisch gesteigerte Zellbildungstätigkeit zur Steig er ung des Reichtums des Blutes an farblosen Blutzellen beisteuern können, sie auch ihrerseits bei primärer Erkrankung eine Leukämie müßten e rze u gen können. Gegen ein solches Vorkommmis habe ich jedoch die so häufigen Fälle geltend gemacht, in welchen aleukämische Tumoren dieser Organe, gleichbeschaffen mit denen der Leukämie, lange Zeit unter dem Bilde einer .Pseudoleukämie" bestehen. Auch die späteren Forschungen haben keine genügenden Aufschlüsse darüber gebracht, wie sich diese Tatsache mit der Annahme einer lienalen oder lymphatischen Leukämie in Einklang bringen läßt.

Was die $\mathrm{Mil} z$ betrifft, so hat dieselbe nach übereinstimmendem Lrteil die ihr von Virch ow zugewiesene hervorragende Stellung in der Leukämielehre nicht wieder zurückerobern können. $\mathrm{Ehrlich}$, welcher ihr für die Erzeugung der farblosen Blutzellen überhaupt nur eine geringe Bedeutung beimißt, will niemals den klinischen Befund einer rein lienalen Form der Leukämie bestätigt gefunden haben.

Anders steht es mit den $\mathrm{L}$ y $\mathrm{m} p \mathrm{~h} d \mathrm{r} \ddot{\mathrm{u}}$ s e $\mathrm{n}$, welche ja nach der herrschenden Lehre für die "lymyphatische Leukämie" auch in den Fällen nachweisbarer Knochenmarksaffektion in erster Linie verantwortlich gemacht werden, und doch steht es yerade für sie fest, daß jhre leukämischen Schwellungszustände sich von denen der Pseudoleukämie nicht unterscheiden lassen. Ehrlich-Pin cus (a. a. O. S. 88) sagen ausdrücklich, daß die pesudoleukämischen Nenbildungen in ihrem histologischen Bau völlig mit denen der lymphatischen Leukämie übereinstimmen, ,in den exstirpierten Drüsen (bei Pseudoleukämie) findet man die Anordnung der normalen Lymphdrüse mit großzelligem Keimzentrum und kleinzelliger peripherischer schicht noch erhalten, ganz so, wie es $\mathrm{Biz}$ z o z e r o für die Leukämie beschrieben“. Wohl sind Versuche gemacht worden, histologische Unterschiede ausfindig zu machen, so wurde von Troje die Ansicht ausgesprochen, daß die leukämischen Lymphome sich dadurch auszeichneten, daß hier eine Durchwucherung der die Follikel trennenden Septen stattfände und damit ein Eintritt der Jymphozyten in die Lymphwege erleichtert würde; B e n d a fand jedoch dieselben Verhältnisse auch bei aleukämischen Lymphomen. Die Verwertung eines von $\mathrm{B}$ e n d a selbst gemachten Befundes, nämlich einer Infiltration der Venenwände bei leukämischen Lymphomen, ist vorläufig nicht angängig, da vergleichende Angaben über die aleukämischen Tumoren fehlen. Bill r o th s einst ausgesprochene Mahnung ${ }^{1}$ ) zur Vorsicht bei der Annahme, daß lymphatische Leukämien aus hyperplastischen Lymphdrüsen hervorgehen, dürfte also immer noch beherzigenswert sein.

1) Billroth, Virch. Arch. Bd. 21, 1861. 
Sollte die von Ehrli c h vertretene Beobachtung, daß auch bei der Pseudoleukämie ein geringer Grad von Lymphozytenvermehrung im Blute, die sich zwar nieht in einer Änderung des. Verhältnisses zwischen roten und farblosen Zellen, wohl aber in einer relativen Zunahme der Lymphozyten im Verhältnis zu den multinukleären Zellen äußert, stets vorhanden ist, daß also Pseudoleukämie und wahre lymphatische Leukämie in bezug auf die Blutbeschaffenheit nur graduell verschieden sind, sich bẹstätigen, so werden wir nach obiger Auseinandersetzung auch diesen graduellen Unterschied nicht auf Rechnung einer durch die pathologischen Veränderungen bedingten Steigerung der Funktion der Lymphdrüsen beziehen dürfen, die Erklärung vielmehr mit viel größerer Wahrscheinlichkeit darin zu suchen haben, daß die für sich bestehenden Drüsenerkrankungen immer nur eine unbeträchtliche Menge von Lymphozyten in das Blut zu werfen vormögen, eine hinzutretende ,lymphoide Metaplasie" des Krochenmarks dieselbe aber so steigert, daß nun erst eine wirkliche Leukämie zustande kommt. Es wird ja nicht geleugnet werden können, daß, wie ich bereits wiederholt hervorgehoben habe, die wuchernden Zellen des in eine starre Knochenwand eingeschlossenen Markes, auch ohne chemotaktische Anlockung, durch Platzmangel gewissermaßen gezwungen sind, einen Ausweg in den Blutstrom zu suchen und sich erst sehr langsam durch Arrosion des Knochens von innen her Platz verschaffen können, während die nachgiebige Lymphdrüsenkapsel eine starke Anhäufung der Zellen zuläßt, ohne daß eine Entlastung durch Ausschwemmung erforderlich wäre.

\section{$\mathrm{Zusammenfas}$ sung.}

1. Nach der Beschaffenheit der Majorität der farblosen Zellen im Blute läßt sich eine ly $\mathrm{phozytäre} \mathrm{Leukämie} \mathrm{(Lymphämie,} \mathrm{Lym} \mathrm{-}$ phozytämie), eine großlymphozytäre (Makrolymphozytämie) und eine myelozytäre Leukämie (Myelozytämie, M y e l ä mi e ) unterscheiden.

2. Die Beschaffenheit der im Blute befindlichen Zellen ist abhängig von dem Charakter des in den blutbildenden Organen ablaufenden hyperplastischen Prozesses und stimmt meist mit der Beschaffenheit der durch diesen an Ort und Stelle gebildeten Zellen überein, ebenso auch mit den Zellen der in andern Organen anftretenden leukämischen Neubildungen. Die Veränderungen der blutbildenden Organe müssen demnach ebenfalls als $1 \mathrm{ymphozytäre,großly} \mathrm{mpho-}$ zytäre und myelozytäre Hyperplasien unterschieden werden.

3. Von welchen Zellen der blutbildenden Organeder zur Hyperplasie führende ProliferationsprozeB aus geht, ist durch die anatomiseh-histologische Untersuchung noch nicht festgestellt; es ist daher unstatthaft, den Ursprung der in den hyperplastischen Blutbildungsorganen bzw. in leukämischen Neubildungen vorhandenen Elemente auf ein bestimmtes System von Zellen, das lymphatische oder das myeloide System Ehrlichs mit Ausschließung anderer 
Zellen zurückzuführen und zwei verschiedene Arten von Leukämien zu unterscheiden, von denen die eine diesem, die andere jenem System entspringt.

4. Am konstantesten betrifft der hyperplastische Prozeß bei der Leukämiedas Knochenmark, und wahrscheinlich sind sämtliche Leukämien myelogenen Ur$\mathrm{sprung}$. Fälle rein lienaler oder rein lymphatischer (aus den Lymphdrüsen stammender) Leukämie sind nicht sicher erwiesen.

5. Die Frage nach der Beteiligung der einzelnen Blutbildungsorgane an der Entstehung der Léukämie isł unabhängig von dem Streite über die Spezifität der Leukozyten und über die Genese der Zellen bei leukämischen Hyperplasien.

\section{XXXIII. \\ Über die Entstehung der Corpora amylacea in der Lunge.}

(Aus dem Pathologisehen Institute der Universität Heidelberg.)

Von

Henning Brütt

aus Hamburg.

(Hierzu Taf. VIII.)

Corpora a m lacea in der Lunge sind zuerst von Friedreich beschrieben worden. Er fand einmal bei brauner Induration, ein andermal bei Kompressionsatelektase, ferner bei einem hämorrhagischen Infarkt konzentrisch geschichtete Körperchen, die teils in den Alveolen, teils im Zwischengewebe lagen. Diese Körperchen gaben die zuerst von $\mathrm{Vir} \mathrm{ch} 0 \mathrm{w}$ bei den Prostatakonkrementen beobachteten Amyloidreaktionen. Außer der konzentrischen Schichtung beobachtete $\mathrm{Fr}$ i edrei $\mathrm{ch}$ oft eine radiäre Streifung sowie fast stets Fremdkörpereinschlüsse, wie Kohlepigment, angeblich auch Kristalle und unregelmäßig gestaltete, klumpige Massen, sowie homogene Kügelchen.

Die Entsteh ung führte er darauf zurück, daß um einen präexistierenden Kern, der meist aus einer der eben erwähnten Substanzen besteht, sich konzentrische Schichten ablagern; diese Schichtsubstanzen, meinte er, würden vom Faserstoff geliefert, während der Blutfarbstoff sich am Aufbau des Kerns beteilige. Eine zelluläre Entstehung der Corpora a m y l a ce a hält er, wie er ausdrücklich betont, für unwahrschéinlich; im Gegensatze zu späteren Forschern, wie $\mathrm{Zahn}$ und $\mathrm{Langhans}$, die fast die gleichen morphologischen Befunde feststellten, dagegen für eine zelluläre Genese eintraten. Erwähnt sei jedoch, daß $\mathrm{L}$ a $\mathrm{ngh}$ a n s in seinem Falle von Lungenkarzinom die Corpora amylacea aus rundlichen Krebszellen entstehen ließ, während $\mathrm{Z}$ a $\mathrm{h} n$ zum ersten Male auf die Entstehung aus desquamierten Alveolarepithelien hinwies. Ferner stellte er fest, daß nicht immer ein Fremdkörper zur Entstehung eines Corpus amylaceum notwendig sei, daß vielmehr kleine amyloide Kügelchen selbst den „Kern“" eines Corpus amylaceum liefern könnten. Eine Bildung der Amyloidkörper im Zwischengewebe hält er für nicht wahrscheinlich; er tritt einzig und allein für die Entstehung der Corpora amylacea in den Alveolen ein.

Auf diesen alten Forschungsergebnissen basieren die Untersuchungen von $\mathrm{Cohn}$, der zum ersten Male eine erschöpfende Darstellung über die Morphologie und Genese der Amyloidkörper gab. Er fand, daß in manchen Fällen, wo der morphologische Befund nicht bezweifeln lieb, daß es sich um Corpora amylacea handele, die charakteristischen Farbreaktionen versagten 